\title{
Continuously-fed gravity currents propagating over a finite porous substrate
}

Cite as: Phys. Fluids 31, 126601 (2019); https://doi.org/10.1063/1.5124955

Submitted: 19 August 2019 . Accepted: 16 November 2019 . Published Online: 11 December 2019

Sara Venuleo (D), Dubravka Pokrajac (D), Anton J. Schleiss, and Mário J. Franca (D)

$\frac{4}{6}$

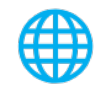




\title{
Continuously-fed gravity currents propagating over a finite porous substrate
}

\author{
Cite as: Phys. Fluids 31, 126601 (2019); doi: 10.1063/1.5124955 \\ Submitted: 19 August 2019 - Accepted: 16 November 2019 • \\ Published Online: 11 December 2019
}

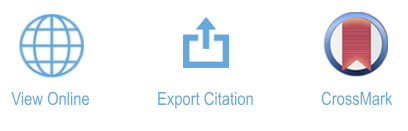

\author{
Sara Venuleo, ${ }^{1, \text { a) }}$ (D) Dubravka Pokrajac, ${ }^{2}$ (D) Anton J. Schleiss, ${ }^{1}$ and Mário J. Franca ${ }^{3}$ (D)
}

\begin{abstract}
AFFILIATIONS
${ }^{1}$ Platforme de Constructions Hydrauliques (PL-LCH), School of Architecture, Civil and Environmental Engineering, École Polytechnique Fédérale de Lausanne (EPFL), Lausanne 1015, Switzerland

${ }^{2}$ School of Engineering, University of Aberdeen, King's College Aberdeen, Aberdeen AB24 3UE, Scotland, United Kingdom

${ }^{3}$ River Basin Development Chair Group, IHE Delft Institute for Water Education and Department of Hydraulic Engineering, Delft University of Technology, Delft, The Netherlands
\end{abstract}

a) Electronic mail: sara.venuleo@epfl.ch

\begin{abstract}
We present the results of laboratory investigations of continuously-fed density currents that propagate first over a smooth horizontal bed and then over a porous substrate of limited length. Inflow discharge, initial excess density, and substrate porosities are varied. Density measurements, acquired through an image analysis technique, are performed above the porous layer simultaneously with quasi-instantaneous vertical velocity profiles. After a first phase in which the current sinks into the substrate, freshwater entrainment from the bed begins and, gradually, a mixing layer forms at the interface between the surface flow and the porous bed. Shear-driven and Rayleigh-Taylor instabilities rule the dynamics of this mixing layer. The porous boundary effects are observed in the vertical distributions of both density and velocity, especially in the near-bed region. Here, larger flow velocities are recorded over porous substrates. We argue that these are due to the presence of a longitudinal pressure gradient, which in turn is a consequence of the current mass loss. Its presence over the porous substrate is proved by the current interface longitudinal slope. However, other effects of the presence of the porous substrate, such as the relaxation of the no-slip boundary condition and the bed-normal momentum exchange, also affect the velocity field. The turbulent structure changes significantly over the porous substrate: while streamwise turbulence decreases, shear and bed-normal Reynolds stresses increase in large part of the current depth. Buoyancy instabilities further enhance the bed-normal momentum flux and, in the near-bed region, contribute to turbulent kinetic energy generation together with shear.
\end{abstract}

Published under license by AIP Publishing. https://doi.org/10.1063/1.5124955

\section{INTRODUCTION}

Gravity currents are geophysical flows occurring when the density difference between two contacting fluids drives their relative motion. They can be observed in an extremely wide range of natural or anthropogenic scenarios, in the atmosphere, in oceans, in lakes, or in industrial processes. A complete classification of these phenomena is given by Simpson. ${ }^{1}$

Human made or natural gravity currents often travel over rough and porous substrates. Several numerical ${ }^{2-5}$ and experimental studies $^{6-12}$ have extensively investigated the bed roughness effects on the structure and dynamics of gravity flows. On the other hand, less attention has been given to density currents traveling over porous beds, which still occur in many situations, often related to human safety and environmental protection. Examples are gravity currents traveling over fissured lake bottoms, oil industry lubricants spilling in a permeable stratum and forming gravity driven flows, ${ }^{13}$ desalinization plant outflows spreading over permeable coastal beds, waste liquids leaking over surrounding granular beds, turbidity currents disrupting deposits of toxic gases, and fluids lying in the porous sea floor. All these flows may entrain, transport, and finally release nutrients, oxygen, as well as dangerous substances into the environment. ${ }^{14,15}$ The prediction of their entrainment and transport capacity requires further studies about their near bed dynamics and turbulence structure.

The first experimental studies of gravity currents traveling over porous beds, consisted of lock-exchange flows propagating over two overlapping metallic grids. ${ }^{16-18}$ Both the cases of viscous ${ }^{17}$ and 
inertial ${ }^{18}$ flows through the porous substrate were investigated. (If the Reynolds number of the flow through the porous milieu is larger than one, the flow is considered to be inertial. Vice versa, it is considered to be viscous.) Their main findings concerned the time evolution of the current front position and mass loss.

Gravity currents over porous boundaries were also studied numerically: two-layer shallow-water models were used to describe the motion of a finite volume of denser fluid into a lighter still one, downward a porous slope, ${ }^{19}$ and over a sloping porous bed in the case of axial symmetry. ${ }^{20}$ Ungarish and Huppert ${ }^{21}$ solved numerically shallow water equations for high Reynolds number density currents spreading over porous substrates, obtaining results in agreement with the experiments of Thomas et al. ${ }^{17}$ In these studies, the porous substrate was considered thin; therefore, only its permeability and the weight of the heavier fluid traveling over it were considered to drive the sinking. If the porous milieu is thick, in addition to the permeability and the weight of the overlying fluid, the weight of the fluid percolated into the substrate has also to be taken into account, as shown by Acton et al. ${ }^{22}$ who investigated analytically and experimentally viscous gravity currents (reproduced releasing a finite volume of glycerin) traveling over a deep layer of spheres. Glass spheres were also used by Thomas et al., ${ }^{23}$ who investigated the behavior of inertial (high Reynolds number) lockexchange gravity flows over a thick porous layer. They arranged glass beads in a submerged container having permeable walls and overlying a free space filled with ambient water. In this way, the fluid penetrating the porous layer could percolate in the water under it. Thomas and Marino ${ }^{15}$ extended their findings studying the same setup but confining the porous layer bed with an impermeable boundary.

Existing numerical and experimental studies of gravity currents flowing over porous substrates mainly deal with lock-exchange flows, which extinguish by losing their mass through the porous milieu over which they travel. Only a few contributions deal with continuously-fed gravity currents, and the existing ones deal with viscous flows propagating over and into porous substrates (Table I by Guo et al. ${ }^{24}$ provides a complete list of these studies). Moreover, research about gravity flows over porous boundaries mainly concerns the front velocity and the current mass loss rate, while little is said about the velocity and density profiles and how the interaction with the porous substrate may alter the turbulence and the mixing occurring at the lower and upper current interfaces. Indeed, for constant density flows, it has been shown that bed permeability can alter significantly the structure and dynamics of turbulence in the near-bed region. ${ }^{25}$

In the present study, we investigate the structure of continuously-fed, inertial gravity currents traveling over a porous substrate, which is located in a limited portion of the domain: $3 \mathrm{~m}$ downstream from the inlet. 25 experiments are carried out varying initial excess density, inflow discharge, and bed porosity. Experimental results are used to

- characterize the flow dynamics at the porous substrate interface: i.e., whether the flow is entering the porous substrate or vice versa whether there is clear water entrainment from the bed;

- investigate how the momentum exchange across the porous substrate interface can alter the mean flow (bulk Froude, bulk Reynolds and gradient Richardson numbers, and density and velocity vertical distributions);

- investigate whether the lower boundary condition (i.e., the presence of the porous substrate) affects the dynamics and the entrainment at the upper current interface. Indeed, it has been shown ${ }^{28}$ that bed roughness can affect the turbulent structures forming at the upper current interface and consequently the freshwater entrainment;

- assess if the presence of the porous substrate is responsible for extra bed resistance;

- observe how the momentum exchange between the surface and subsurface flow alters the turbulence structure of the surface flow.

This paper is structured as follows: in Sec. II, the facility used to perform the experiments is described together with the porous substrate and the instrumentation and techniques used to acquire density and velocity measures; the experimental program is also introduced in Sec. II; Sec. III presents the experimental results, starting with the mean flow description followed by the characterization of the turbulent structure; and finally in Sec. IV, results are summarized and discussed.

\section{MATERIALS AND METHODS}

\section{A. Installation and experimental procedure}

Experiments were carried out in a $5.5 \mathrm{~m}$ long, $0.45 \mathrm{~m}$ deep, $0.275 \mathrm{~m}$ wide horizontal flume with transparent Plexiglas side walls. Before each experiment, a brine mixture of desired density is carefully prepared in a $2 \mathrm{~m}^{3}$ tank, by mixing freshwater with a known amount of salt and ink. The latter is fundamental to measure the flow density through an image analysis technique, which will be explained in detail in Sec. II C. The homogeneity of the prepared solution is achieved using a low-head pump, submerged in the tank, where it recirculates the fluid during the experiment preparation and execution.

A pumping circuit connects the mentioned tank to an automatic pump and the latter to the upstream part of the channel (see Fig. 1). Here, an inlet structure with a diffuser ensures uniformly distributed inflow velocity and density. Inlet discharge is kept constant by a PID (Proportional- Integral-Derivative) control, implemented in LabView, which adjusts the automatic pump power to minimize the difference between the chosen target inflow and the instantaneous circuit discharge. This is continuously monitored by a flowmeter downstream from the pump. Once the pump is started, a gravity current forms and travels for $3 \mathrm{~m}$ over a smooth horizontal bed before reaching the porous substrate. Note that due to the horizontal bed, these currents cannot reach normal (uniform) flow conditions.

A false bed, placed $0.2 \mathrm{~m}$ above the original one, covers the entire channel except where the porous substrate is placed (in $1 \mathrm{~m}$ long cavity). Our measuring window is located here at the transition between the nonporous and porous bed so that part of both is monitored by the camera.

Downstream from the porous substrate, the current travels again over a smooth horizontal bed, until it reaches the downstream end of the channel, where it plunges to a $12 \mathrm{~m}^{3}$ tank without being 

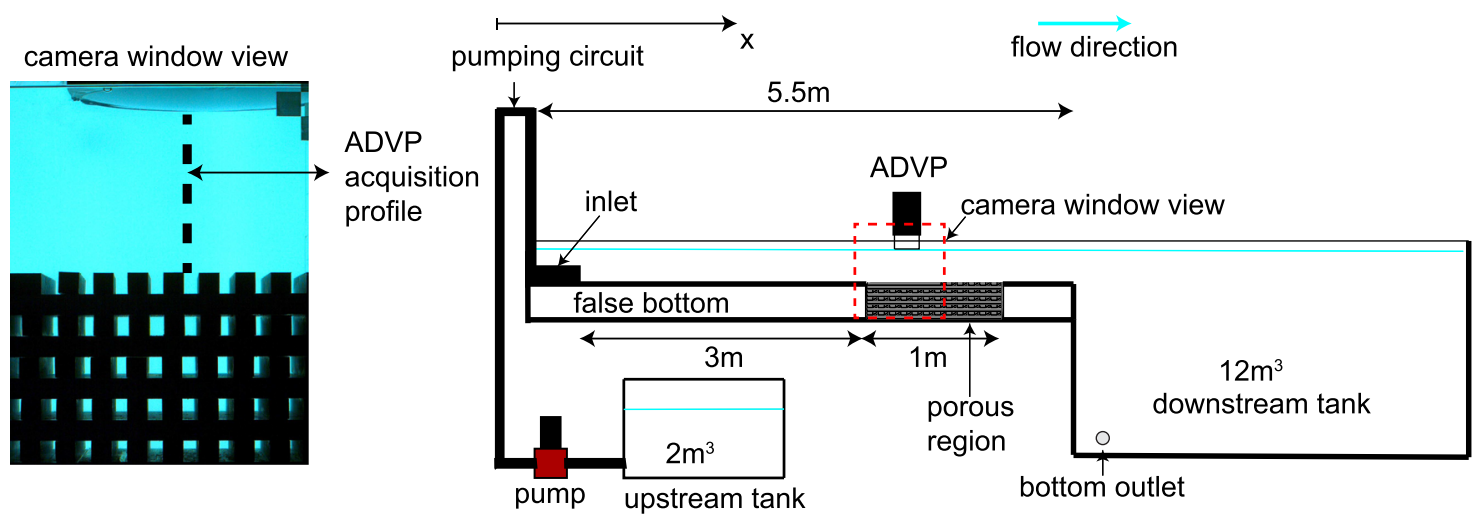

FIG. 1. Sketch of the experimental facility and location of the measuring window.

reflected backward. Right after the pump is started, the downstream tank outlet is opened. A valve allows regulating the out-flowing discharge to release the same volume injected upstream and keep the water level in the whole system constant. An ultrasonic water level sensor, located immediately downstream the porous substrate, allows monitoring level fluctuations. Some small waves are observed at the very beginning of the experiment due to the sudden increase in the pump discharge but fade once the current starts to form and advance (becoming less than $0.5 \mathrm{~mm}$ fluctuations).

\section{B. Porous substrate}

A false bed covers the entire channel length except for a $1 \mathrm{~m}$ long section starting at $3 \mathrm{~m}$ downstream the inlet, where the real bed is left uncovered, creating a $0.2 \mathrm{~m}$ deep and $0.275 \mathrm{~m}$ wide cavity. Here, we built a porous substrate by placing $20 \mathrm{~mm} \times 20 \mathrm{~mm}$ PVC bars in crossing layers. Varying the spacing between consecutive PVC bars, we obtained substrates of different porosities. All porous bed arrangements were made up of five layers (to obtain a total porous substrate depth of $20 \mathrm{~cm}$ ), each composed of one cross-stream and one streamwise placed series of bars (see Fig. 2). This particular disposition was chosen to allow density visualization inside the porous substrate cavities.

Table I summarizes the characteristic lengths of the porous substrates: the spacing between consecutive PVC squared bars $s$, their side $d$, their length $l_{C S W}$ (when placed in the cross-stream direction), and their length $l_{S W}$ (when placed in the streamwise direction).

The downstream end of the porous substrate is connected to the space underlying the false bed, which is submerged and therefore filled with ambient water. This configuration, hereafter referred to as "non-confined," allows the water within the substrate cavities to leave the porous layer under the false bed. Few experiments were performed after filling the porous substrate cavities with the same brine solution used to feed the current. This configuration is hereafter called "filled."

\section{Density measurements}

2D (two-dimensional) instantaneous density measurements are performed applying an image analysis technique based on inklight absorption similar to the one applied by Nogueira et al. ${ }^{30}$ or
Balasubramanian et al. $^{31}$ A light-emitting diode (LED) panel is placed on one side of the channel to back light the flow in the camera recording window. On the opposite side, a Basler CMOSIS color camera is placed to acquire flow images.

The ink chosen to carry out our experiments is fluorescein sodium salt: a fluorescent dye absorbing wavelengths in the range of $485-495 \mathrm{~nm}$ (blue) and emitting wavelengths in the range of 520-525 nm (green). This property allows us to derive a relation between ink concentration and the blue value of the pixels. A careful calibration procedure was followed to identify the range in which the relation blue value-ink concentration was expressed by a monotonic function, to obtain for each pixel a blue value-ink concentration calibration curve. A pixel by pixel calibration allows us to get rid of spurious effects due to possible light non-uniformity. During the calibration and the experiment, constant lighting conditions are ensured by an opaque black curtain that completely covers the channel in the region of the camera measuring window.

Before the experiment starts, a chosen amount of ink is added to the brine water prepared in the tank upstream the injection pump. Here, the homogeneity of the solution is achieved employing a low head submerged pump, which recirculates the fluid before and during the experiment. Once this brine water of known density and known ink concentration is injected into the channel, it mixes with fresh ambient water, forming the gravity current. Flow images are recorded and their blue color is used to compute the width-averaged ink concentration map by applying, pixel by pixel, the mentioned derived calibration curve. Finally, as fluorescein is a passive tracer, its width-averaged concentration is used to estimate the width-averaged density map considering that salt and ink concentrations are proportional. Figure 3 summarizes the steps of the image analysis technique applying it to one image from experiment D1020_Q13_P20. In the plot on the right, the white line marks the current interface, which is identified as the location where the excess density becomes lower than $10 \%$ of the initial excess density $\Delta \rho_{I}$.

\section{Velocity measurements}

A three-dimensional quasi-instantaneous velocity profile is acquired over the porous substrate by means of an Acoustic Doppler 

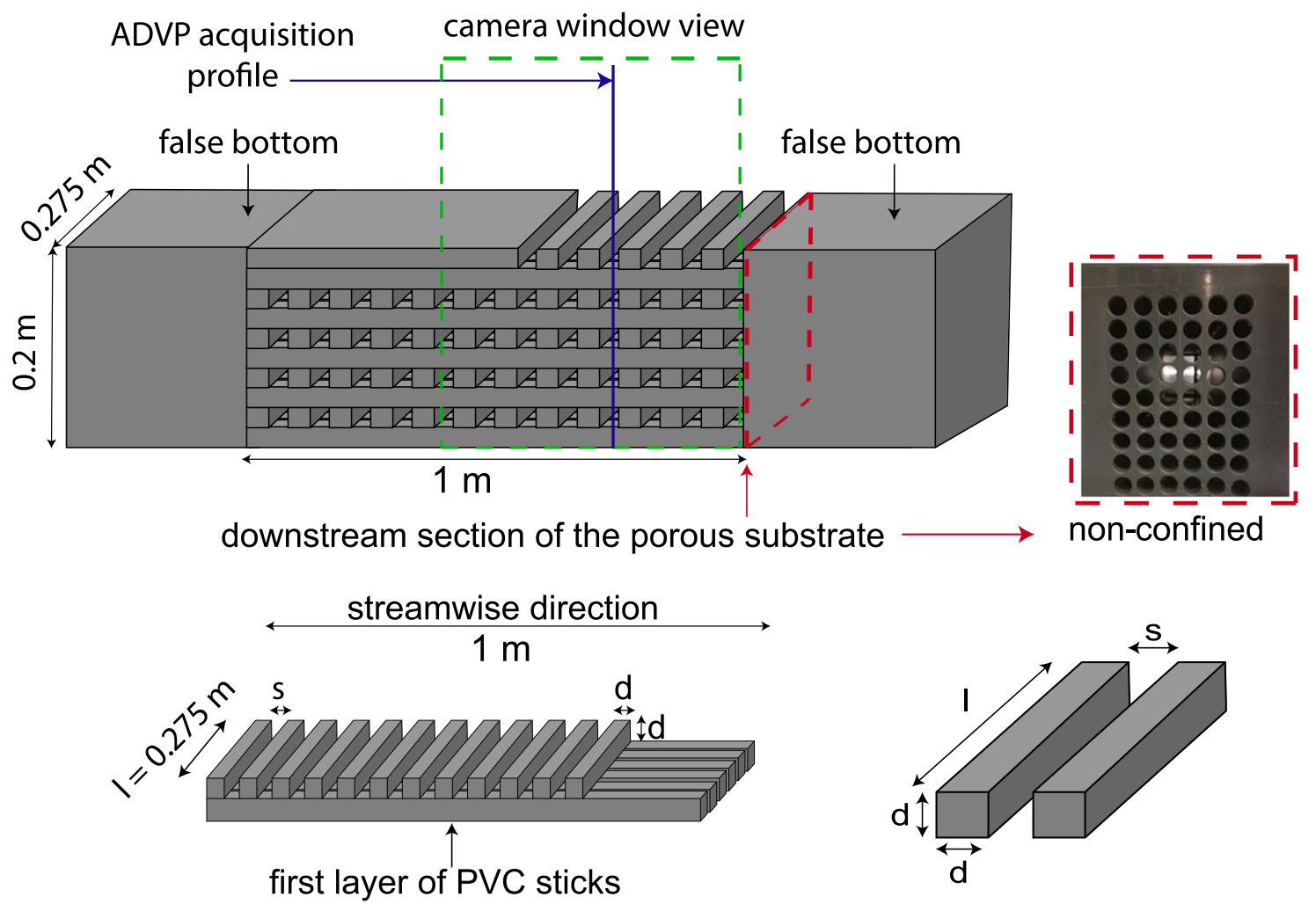

FIG. 2. Sketch of the porous substrate, structure of one of its layers, and characteristic lengths of the PVC squared bars.

Velocimetry Profiler (ADVP). The working principle of this acoustic sonar instrument is presented in detail by Blanckaert and Lem$\min ^{32}$ and Franca and Lemmin, ${ }^{33}$ among others, and consists of measuring the frequency-shift of the emitted acoustic signal, which is induced by targets moving with the flow. The ADVP used in our experiments was developed at EPFL (École polytechnique fédérale de Lausanne) and consists of one emitter and four receivers, whose placement allows us to obtain one redundant estimation for each velocity component and consequently to reduce the noise level in the measurements. ${ }^{32}$

Velocity profiles are acquired in one vertical in the middle of the porous substrate $(18 \mathrm{~cm}$ downstream the first cavity, see Fig. 3 )

TABLE I. Porous bed characteristics lengths: the spacing between consecutive PVC squared bars $s$, their side $d$, bed cavity aspect ratio $s / d$, bars length when placed in the cross-stream direction, $I_{C S W}$, and bar length when placed in the streamwise direction, $I_{S W}$.

\begin{tabular}{lccccc}
\hline \hline Porosity & $\mathrm{s}(\mathrm{mm})$ & $\mathrm{d}(\mathrm{mm})$ & $s / d$ & $l_{C S W}(\mathrm{~mm})$ & $l_{S W}(\mathrm{~mm})$ \\
\hline 0.00 & 0.0 & 20 & 0.000 & 275 & 1000 \\
0.12 & 2.5 & 20 & 0.125 & 275 & 1000 \\
0.20 & 5.4 & 20 & 0.270 & 275 & 1000 \\
0.29 & 8.2 & 20 & 0.410 & 275 & 1000 \\
0.42 & 15.0 & 20 & 0.750 & 275 & 1000 \\
\hline \hline
\end{tabular}

with frequency $31.25 \mathrm{~Hz}$. The ADVP acquisition is triggered by the rising edge of a square electrical signal generated by a function signal generator. The same electrical signal is used to trigger the camera shooting, allowing us to synchronize velocity and density measures.

Acoustic targets are introduced in the flow at the inlet location and consist of microhydrogen bubbles generated by an electrolysis process according to the technique of Blanckaert and Lemmin, ${ }^{32}$ who showed that a high acoustic scattering level has to be achieved in order to measure turbulence with sufficient accuracy. The size of these bubbles was sufficiently small (less than one pixel) to assume they do not interfere with the image analysis.

To further decrease the measurement noise level, the following cleaning steps were applied: prior to velocity computations, the raw frequencies acquired from the ADVP were filtered using a Matlab subroutine written by Mori ${ }^{56}$ based on the method proposed by Goring and Nikora; ${ }^{34}$ afterward, the Matlab built in function medfilt 2 was applied to the velocity field: this function performs $2 \mathrm{D}$ median filtering so that each velocity value is the median value of its $\mathrm{m} \mathrm{x} \mathrm{n}$ neighborhood in the original velocity field. The chosen size of the neighborhood cell was $3 \times 3$. Remaining outliers were identified as the values that differ more than three standard deviations from the mean of the surrounding $3 \times 3$ block of cells. They were replaced by the mean of the block. Finally, a low pass filter was designed in Matlab to cut energy at frequencies higher than $10 \mathrm{~Hz}$. The algorithm "filtfilt" implemented in Matlab 

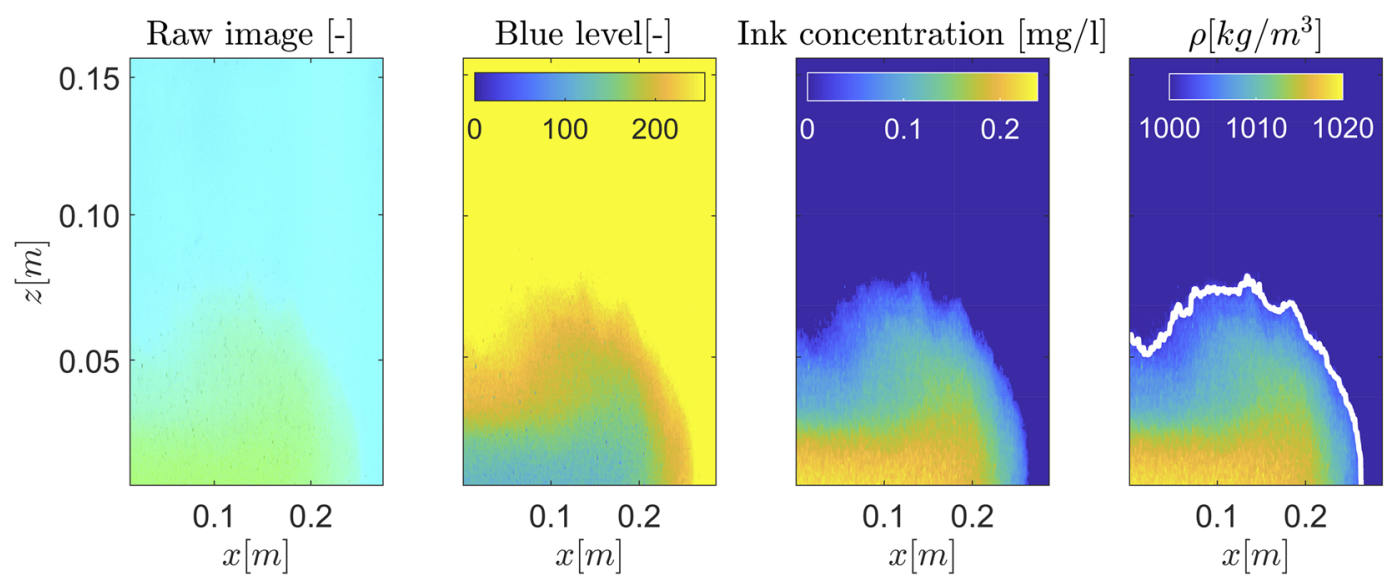

FIG. 3. Steps of the image analysis technique used to estimate the width-averaged density field. From left to right: the raw acquired image of the head of the density current, its blue level, the corresponding width averaged ink concentration, and density field. In the plot on the right, the white line marks the current interface identified with a density threshold method. Data shown concern experiment D1020_Q13_P20.

allowed the low pass filtering of the data with zero-phase distortion. Figure 4 shows the longitudinal, $u$, and vertical, $w$, velocity field after noise-cleaning together with the density time evolution at the ADVP location.

\section{E. Experimental program}

Twenty five experiments were carried out varying three main variables: initial density $\rho_{I}$, inflow discharge $Q$, and substrate porosity $\phi$. Experiments are named with reference to these three main parameters: "D," an abbreviation for "density," is followed by the initial density of the experiment; "Q," the inlet discharge, is followed by the inflow discharge expressed in deciliter per second; and "P," an abbreviation for "porosity," is followed by the percentage of the void volume.

Experiments are listed in Table II, where according to the initial conditions ( $\rho_{I}$ and $\left.Q\right)$, they are grouped in four groups: group 1 (GR1), group 2 (GR2), and group 3 (GR3) are characterized by the same initial density $\left(\rho_{I}=1006\right)$ but varying inlet discharge, equal to $0.7 \mathrm{l} / \mathrm{s}, 1.2 \mathrm{l} / \mathrm{s}$, and $1.4 \mathrm{l} / \mathrm{s}$, respectively. Group 4 (GR4) is
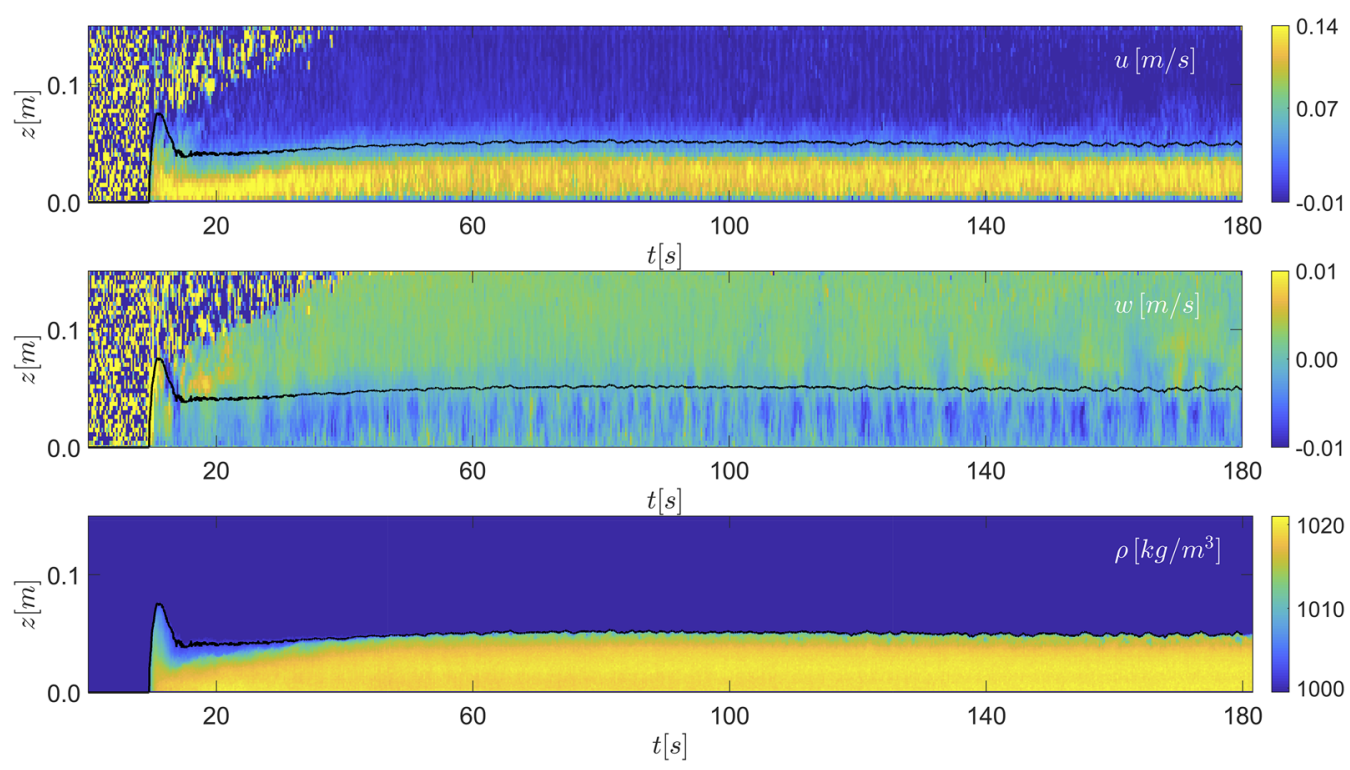

FIG. 4. Example of data collected for each experiment: from top to bottom, time evolution of the longitudinal velocity, $u$, the vertical velocity, $w$, and the density, $\rho$, at the ADVP location. The experiment shown is D1020_Q13_P29. The black line identifies the current interface defined applying a density threshold method. 
TABLE II. Experiments list and main parameters. The flume slope is zero for all the listed experiments.

\begin{tabular}{|c|c|c|c|c|c|c|c|}
\hline Group & Name experiment & $\mathrm{Q}(\mathrm{l} / \mathrm{s})$ & $\begin{array}{c}\text { Initial density } \\
\rho_{I}\left(\mathrm{~kg} / \mathrm{m}^{3}\right)\end{array}$ & Porosity $\phi$ & Filling & $F r_{I}$ & $\operatorname{Re}_{I}$ \\
\hline \multirow{6}{*}{1} & D1006_Q07_P00 & \multirow{6}{*}{0.7} & \multirow{6}{*}{1006} & 0 & & \multirow{6}{*}{0.74} & \multirow{6}{*}{2355} \\
\hline & D1006_Q07_P12 & & & 0.12 & Ambient fluid & & \\
\hline & D1006_Q07_P20 & & & 0.2 & Ambient fluid & & \\
\hline & D1006_Q07_P29 & & & 0.29 & Ambient fluid & & \\
\hline & D1006_Q07_P42 & & & 0.42 & Ambient fluid & & \\
\hline & D1006_Q07_P42F & & & 0.42 & Dense fluid & & \\
\hline \multirow{6}{*}{2} & D1006_Q12_P00 & \multirow{6}{*}{1.2} & \multirow{6}{*}{1006} & 0 & & \multirow{6}{*}{1.27} & \multirow{6}{*}{4037} \\
\hline & D1006_Q12_P12 & & & 0.12 & Ambient fluid & & \\
\hline & D1006_Q12_P20 & & & 0.2 & Ambient fluid & & \\
\hline & D1006_Q12_P29 & & & 0.29 & Ambient fluid & & \\
\hline & D1006_Q12_P42 & & & 0.42 & Ambient fluid & & \\
\hline & D1006_Q12_P42F & & & 0.42 & Dense fluid & & \\
\hline \multirow{9}{*}{3} & D1006_Q14_P00 & \multirow{9}{*}{1.4} & \multirow{9}{*}{1006} & 0 & & \multirow{9}{*}{1.48} & \multirow{9}{*}{4710} \\
\hline & D1006_Q14_P12 & & & 0.12 & Ambient fluid & & \\
\hline & D1006_Q14_P20 & & & 0.2 & Ambient fluid & & \\
\hline & D1006_Q14_P29 & & & 0.29 & Ambient fluid & & \\
\hline & D1006_Q14_P42 & & & 0.42 & Ambient fluid & & \\
\hline & D1006_Q14_P29F & & & 0.29 & Dense fluid & & \\
\hline & D1006_Q14_P42F & & & 0.42 & Dense fluid & & \\
\hline & D1006_Q14_P42I & & & 0.42 & Ambient fluid $^{\mathrm{a}}$ & & \\
\hline & D1006_Q14_P42IF & & & 0.42 & Dense fluid $^{\mathrm{a}}$ & & \\
\hline \multirow{6}{*}{4} & D1020_Q13_P00 & \multirow{6}{*}{1.3} & \multirow{6}{*}{1020} & 0 & & \multirow{6}{*}{0.76} & \multirow{6}{*}{4322} \\
\hline & D1020_Q13_P12 & & & 0.12 & Ambient fluid & & \\
\hline & D1020_Q13_P20 & & & 0.2 & Ambient fluid & & \\
\hline & D1020_Q13_P29 & & & 0.29 & Ambient fluid & & \\
\hline & D1020_Q13_P42 & & & 0.42 & Ambient fluid & & \\
\hline & D1020_Q13_P42F & & & 0.42 & Dense fluid & & \\
\hline
\end{tabular}

${ }^{\text {a }}$ These experiments were carried out for flow visualization purposes, adding potassium permanganate (purple ink) to the fluid inside the bed cavities.

characterized by the initial density $\left(\rho_{I}=1020 \mathrm{~kg} / \mathrm{m}^{3}\right)$ and inlet discharge $Q=1.3 \mathrm{l} / \mathrm{s}$. In the following, when comparing experiments belonging to the same group, only the last part of the name (i.e., the part of the name characterizing the porosity of the substrate) is used to distinguish them.

The column entitled "Filling" in Table II specifies how the void volume of the porous substrate is filled prior to each experiment: in standard experiments, ambient water (having density $\rho_{0}=1000 \mathrm{~kg} / \mathrm{m}^{3}$ ) fills the void, but for few experiments, brine fluid having density equal to $\rho_{I}$ is carefully injected into the porous substrate replacing the ambient water in its voids. Moreover, two experiments (D1006_Q14_P42I and D1006_Q14_P42IF) were carried out for flow visualization purposes adding potassium permanganate (a purple ink) to the water lying in the porous substrate cavities.

Table II also shows the inlet Froude $F r_{I}$ and Reynolds $R e_{I}$ number of the groups, respectively, computed as $F r_{I}=Q /\left(B_{I} H_{I}\right) / \sqrt{g^{\prime} H_{I}}$ and $R e_{I}=\rho_{I} H_{I} Q /\left(B_{I} H_{I}\right) / \mu$, where $H_{I}$ is the inlet section height, $B_{I}$ is the inlet section width, $g^{\prime}$ is the reduced gravity computed using the initial density $\rho_{I}:\left(g^{\prime}=g\left(\rho_{I}-\rho_{0}\right) / \rho_{0}\right)$, and $\mu$ is the dynamic viscosity of the brine solution.

\section{RESULTS AND DISCUSSION}

\section{A. Bulk flow motion}

\section{Current depth definition}

In the following, the bulk flow motion is described by looking at the main depth-averaged flow variables. The current depth, $h_{c}$, is defined by applying a density threshold definition (as in the work of Ottolenghi et al. $\left.{ }^{35}\right)$ : the fluid having density $10 \%\left(\rho_{I}-\rho_{0}\right)$ higher than the ambient water density is considered to be part of the current. Depth-averaged variables are obtained by applying the general definition,

$$
\psi_{h_{c}}=\frac{1}{h_{c}} \int_{0}^{z^{I}} \psi d z
$$


where $\psi$ is the variable to be depth-averaged and $z^{I}$ is the $z$ coordinate of the current upper interface (i.e., $z_{I}=h_{c}$ ).

\section{Flow phases}

After the head transition, continuously-fed gravity currents propagating over sloping boundaries reach an equilibrium condition also known as a normal condition. ${ }^{8,29}$ In this state, the flow properties are characterized by small gradients in the downstream direction. In our experiments, due to the horizontal bed, normal flow conditions cannot be achieved. ${ }^{29}$ Three main flow phases can be distinguished: a sinking phase, a transition phase, and a final phase, called quasi-steady phase, during which the main bulk flow variables remain quasiconstant in time. These three time phases are observed in Fig. 5(a), which shows the density evolution at the ADVP profile when the bed is filled with freshwater inked with potassium permanganate.

The "sinking phase" is considered to start with the arrival of the current front at the porous substrate location. Here, the current continues advancing and starts sinking into the substrate. The mass loss causes a sharp drop of the current depth. Because continuity has to be satisfied, a volume equivalent to the volume of denser fluid infiltrating into the substrate has to simultaneously leave the substrate. We observed that ahead the front, the ambient fluid laying in the substrate escapes it [see Figs. 9(a) and $9(\mathrm{e})$ ]. However, we cannot exclude the presence of an outflow under the false bed, at the downstream unconfined section of the porous substrate. During the sinking phase, little or no freshwater entrainment from the bed into the current is observed [see Fig. 5(a)].

The "transition phase" is considered to start when the current has reached the final section of the porous substrate $\left(t=t^{*}\right)$. During this phase freshwater entrainment from the bed initiates and the current depth $h_{c}$ starts to increase [see Fig. 5(a)].

Finally, the beginning of the quasi-steady phase corresponds to the time step at which main bulk flow variables (i.e., current depth, depth-averaged density, and depth-averaged velocity) reach an almost constant value in time [see Fig. 5(b)].

\section{Head of the current}

Previous experimental ${ }^{10,36}$ and numerical ${ }^{37}$ studies observed that the advancement velocity of the head of continuously-fed density currents depends on the initial excess density, $\rho_{I}$, and on the inflow discharge per unit of width, $q$, according to

$$
U_{\text {head }}=C\left(g^{\prime} q\right)^{1 / 3},
$$

where $g^{\prime}$ is the reduced gravity and $C$ is a proportionality constant that was found to vary in the range $C=1.05 \pm 0.1$. $^{3}$

The constant which best represents the head velocity of our experimental data is $C=0.93$, which is close to but lower than the values falling in the predicted range. On the other hand, Britter and Linden ${ }^{36}$ reported that for very small slopes (less than $0.5^{\circ}$ ), the head decelerates with the distance from the inlet. As our flume slope is zero, we can expect a front deceleration, which can explain why $C$ is lower than the values predicted in Ref. 38. Moreover, our porous substrate is rough, and this may cause a further front velocity decrease. Although it does not apply for all the experiment groups, the front velocity over porous substrates is in general lower than over nonporous substrates. The observed velocity difference is small (few millimeters per second) and does not depend on the substrate porosity. During the head transition, no relevant entrainment from the bed and therefore no significant head dilution is observed.

We also investigated the dependence of the head maximum height, head length (i.e., time lag between the head arrival and the occurrence of the first local minimum after it), and current-depth height-drop (i.e., difference between the head maximum and the height at the rear of the head) on the substrate porosity: no significant trend could be pointed out. A possible explanation for the lack of any significant differences between the head of currents, traveling on impervious and on porous substrates, may be the low longitudinal extension of the porous substrate in our experimental setup.

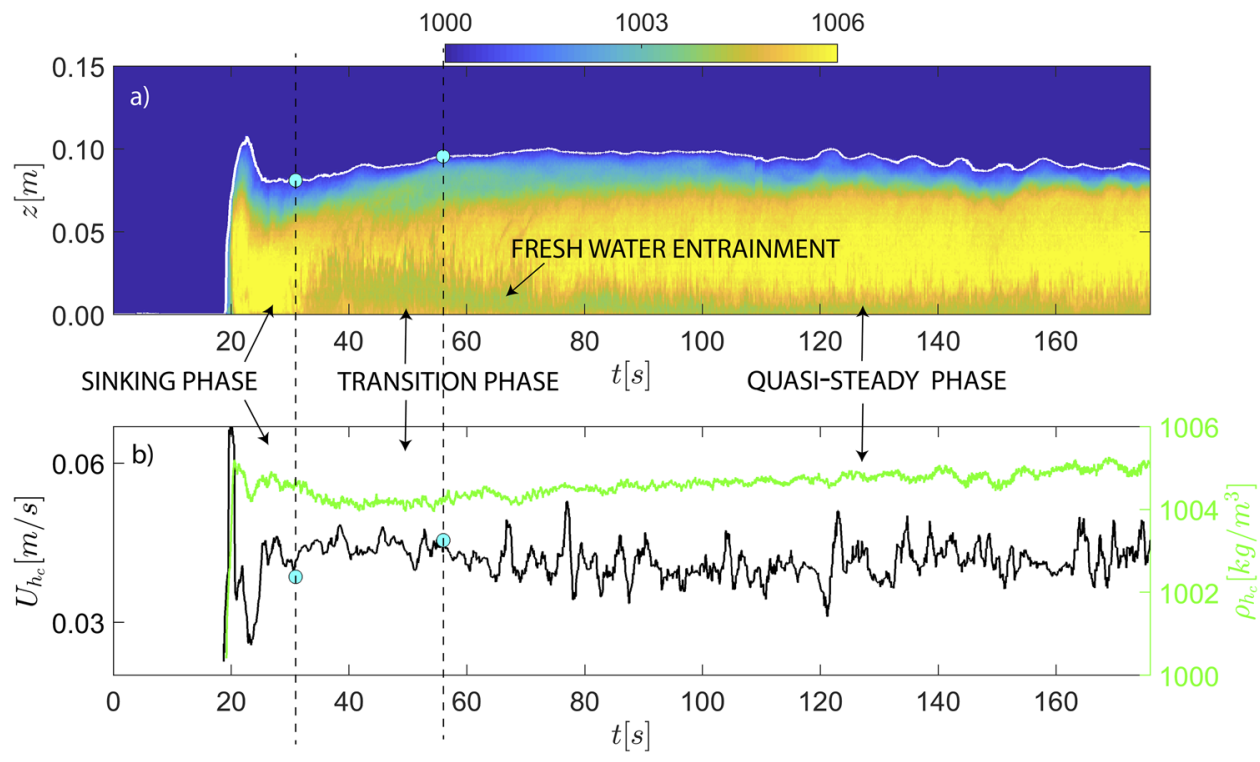

FIG. 5. (a) Density evolution at the ADVP profile when the bed is filled with freshwater inked with potassium permanganate (experiment D1006_Q14_P42/) The white line shows the time evolution of current depth, averaged over the length of the porous material. (b) Time evolution of depth-averaged velocity (black) and density (green) at the ADVP location. Cyan dots identify $t=t^{*}$ and $t=t_{\text {steady }}$ : the beginning of the transition phase and beginning of the quasi-steady phase, respectively. 
However, it should be considered that previous results from lockexchange experiments over porous substrates (where no additional momentum is provided to the current ${ }^{18,23}$ showed that the longitudinal momentum of the current head is not significantly affected by the mass leakage through the bed. This also seems to apply to our case, as the continuous injection of the momentum from the inlet source, even for the larger tested porosities, compensates the mass loss through the bed.

\section{Current depth and interface slope}

Larger porosities are expected to result in faster sinking and therefore in lower current depths. However, this is true only during the first sinking phase. Conversely, if time-averaged during the quasi-steady state of the flow, the current depth observed for porosities $\phi=0.29$ and $\phi=0.42$ is slightly larger than the one observed for porosity $\phi=0.2$ [see Fig. 6(b)]. On the other hand, for porosities lower than $\phi=0.2$, Fig. 6 (b) shows that the thickness of the current decreases with increasing porosity. This result holds independently of the initial inflow conditions, represented by the four groups (see Table II). A possible explanation for the lack of a monotonic trend may be the superimposed effects of the current-sinking and entrainment of bottom freshwater, which feeds the current body volume, increasing its buoyancy. In fact, the higher the substrate porosity, the stronger the mass loss and the sinking, but at the same time, the larger the mass exchange and thereby the entrainment occurring at the porous substrate interface. Experiments performed over porous but filled substrates confirm this explanation: they show flow-thicknesses comparable to the experiments performed over impervious beds since no sinking occurs in both these configurations.

Observing the current interface during the experiment execution, we noted that over the porous substrate reach, the current interface showed a significant slope, which varies with the substrate porosity. With reference to Fig. 6(a), the current interface slope can be computed as

$$
J_{h_{c}}=\frac{h_{2}-h_{1}}{x_{2}-x_{1}},
$$

where $h_{2}$ and $h_{1}$ are the time-averaged (during the steady state) values of current depth in sections 1 and 2, and $x_{1}$ and $x_{2}$ are the corresponding longitudinal locations. Negative slopes imply that $h_{c}$ is decreasing in the flow direction, whereas positive values of $J_{h_{c}}$ imply that the current depth is increasing in the flow direction.

Figure 6(c) shows the time-averaged (during quasi-steady period) slope and its standard deviation. Although the slope time variability is large (standard deviation bars are large), it stands out that $J_{h_{c}}$ changes significantly when the substrate porosity varies: it is positive over flat and filled beds, and negative over porous
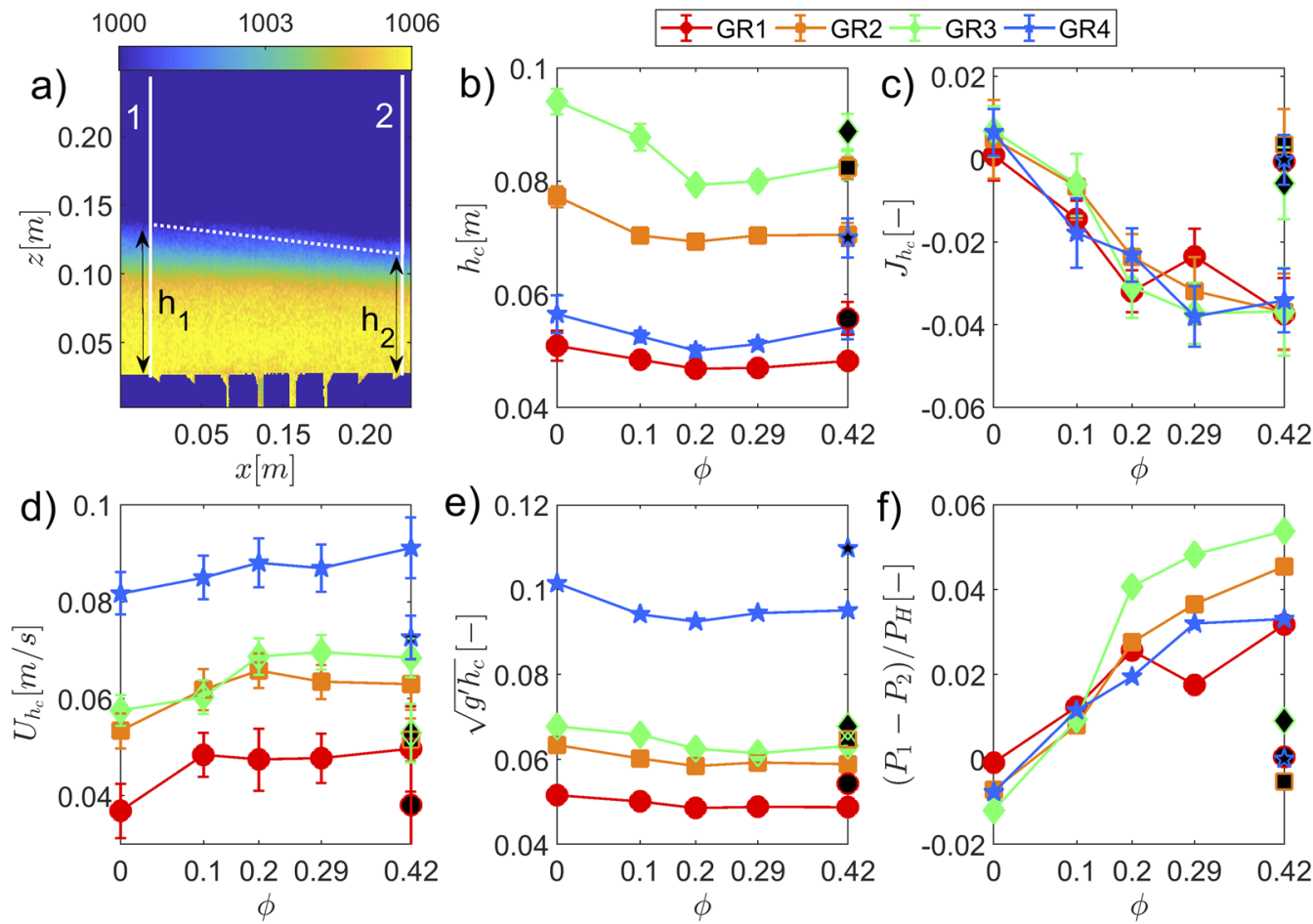

FIG. 6. (a) The reference window, where the spatial average is performed, and sections 1 and 2 used to compute the longitudinal slope. (b) Space (between sections 1 and 2 ) and time (during quasi-steady period) averaged current depth values together with their standard deviation. (c) Time-averaged (during quasi-steady period) longitudinal slope of the current interface and its standard deviation. (d) Time-averaged (during quasi-steady period) depth-averaged velocities. (e) Time and space averaged (during quasi-steady period) buoyancy velocity. (f) Difference between pressure force acting on section $1\left(P_{1}\right)$ and on section $2\left(P_{2}\right)$, normalized by the pressure force of the ambient water column $\left(P_{H}\right)$. Markers filled in black refer to "filled" experiments. 
substrates. In the latter case, it monotonically decreases with growing porosity. These observations suggest that the current interface slope is a consequence of the current mass loss, which occurs over porous substrates. Therefore, the porous substrate represents a sink for the current, which loses mass and energy while advancing on it.

\section{Depth-averaged variables}

To compare the bulk flow over substrates of different porosities, we plotted the depth-averaged [Fig. 6(d)] and buoyancy [Fig. 6(e)] flow velocities, time-averaged during the steady phase. Buoyancy velocities show the same trend observed for current depth values: they diminish with growing substrate porosity, reaching a minimum for $\phi=0.2$ or $\phi=0.29$ (depending on the dataset considered), and slightly increasing over larger substrate porosity. This nonmonotonic trend is due to two opposite effects: the current-sinking, which results in lower current depths, and the bottom freshwater entrainment, which increases the current buoyancy, decreasing its density.

Depth-averaged velocities increase monotonically with substrate porosity. This trend characterizes GR1, GR3, and GR4 experiments, while GR2 data show the maximum depth-averaged velocity for substrate porosity equal to 0.2 . On the other hand, the lowest depth-averaged velocities are observed for currents moving over substrates filled with denser fluid. This suggests that the flow over porous substrates is faster when the current sinks into the substrate.

Moreover, Figs. 6(d) and 6(c) show that the depth-averaged velocities and the current interface slopes are correlated: larger slopes correspond to faster flows and higher porosity values. An explanation for the trend shown by depth-averaged flow velocities can be deduced from this observation: the current interface slope, stemming from the current mass loss, causes the formation of a longitudinal pressure gradient, which in turn results in larger flow velocities. To prove it, we computed the difference between the pressure force acting on the section upstream and on the section downstream the porous substrate, which is shown in Fig. 6(f), normalized by the pressure force of the ambient water column. It turns out that a pressure force gradient exists and increases with growing substrate porosity. However, as will be discussed in Sec. III B 2, other consequences of the presence of the porous substrate can affect the flow resistance and thereby its velocity.

\section{Characteristic dimensionless current numbers}

Table III lists characteristic parameters and dimensionless numbers of the experiments.

The surface flow regime is characterized by the bulk Reynolds and Froude number of the current, $R e_{b}$ and $F r_{b}$, respectively, defined as

$$
\operatorname{Re}_{b}=\frac{h_{c} U_{h_{c}} \rho_{h_{c}}}{\mu}
$$

and

$$
F r_{b}=\frac{U_{h_{c}}}{\sqrt{g^{\prime} h_{c}}},
$$

where $U_{h_{c}}$ is the time-averaged, depth-averaged velocity, $\rho_{h_{c}}$ is the time-averaged, depth-averaged density, and $g^{\prime}$ is the reduced gravity computed using $\left.\rho_{h_{c}}: g^{\prime}=g\left(\rho_{h_{c}}-\rho_{0}\right) / \rho_{0}\right)$.

It can be noted that the Froude numbers listed in Table III are in general lower or slightly larger than one. Considering that the critical Froude number of conservative gravity currents may be larger than the unity; ${ }^{39}$ it can be concluded that the flow regime is generally subcritical. This result confirms the finding of Sequeiros, ${ }^{29}$ who reviewed 78 previous studies and observed that gravity currents propagating over horizontal slopes are characterized by subcritical regimes.

Finally, the Froude number over porous boundaries is larger than over non-porous boundaries. This is a consequence of the simultaneous increase in depth-averaged velocities and decrease in depth-averaged density (and thus of the term $\sqrt{g^{\prime} h_{c}}$ ) over porous boundaries.

$R e_{b u l k}$ values reported in Table III show that the flow regime is fully turbulent for all experiments, excluding few exceptions belonging to group 1 . In general, $R e_{b u l k}$ slightly increases with porosity.

Previous studies about constant-density flow over porous boundaries ${ }^{25-27}$ quantified the substrate porosity effect using the permeability Reynolds number, $R e_{k}$, defined as

$$
R e_{k}=\frac{\sqrt{k} U^{*}}{v},
$$

where $k$ is the bed permeability, $U^{*}$ is the friction velocity, and $v$ is the kinematic viscosity of the flow. In our case, a direct estimate of the bed permeability was not available; therefore, to characterize the flow inside the porous layers, we computed the pore Reynolds number using as the length scale the spacing, $s$, between consecutive PVC squared bars,

$$
R e_{p}=\frac{s U^{*}}{v}
$$

The obtained values of $R e_{p}$ (see Table III) fall in the range of 8-94; therefore, the flow inside the porous substrate is non-Darcian (i.e., the resistance law for the vertical flow through the porous substrate is nonlinear). Indeed, eddies and tortuous streamlines can be observed in the recorded images.

Over natural rough beds, the characteristic length scale, $d$, has to be taken into account to define the roughness effect. In the present case, the roughness Reynolds number, $R e_{d}$, can be defined as

$$
R e_{d}=\frac{d U^{*}}{v}
$$

where $d$ is the height of the first-layer of the PVC bars. Resulting $R e_{d}$ values are comparable with the obtained values of $R e_{p}$. This implies that we cannot exclude roughness effects in our observations.

To classify our roughness, we considered the ratio between the rod spacing, $s$, and height, $d$ (see Table I) (although a more accurate classification should be based on the relative magnitude of frictional and pressure $\left.\operatorname{drag}^{40}\right)$. All our experiments are characterized by a ratio $s / d$ lower than one, and thereby, our roughness can be classified as "d-type" (i.e., recirculation vortices form within the substrate cavities and there is no eddy shedding into the flow above the PVC $\operatorname{bars}^{40}$ ). 
TABLE III. Characteristic parameters and dimensionless numbers of the experiments: the depth-averaged and time-averaged velocity $U_{h_{c}}$, the depth-averaged and timeaveraged density $\rho_{h_{c}}$, the time-averaged current depth $h_{c}$, the bulk current Reynolds number $R e_{b}$, the bulk current Froude number $F r_{b}$, the friction velocity $U^{*}$, the maximum velocity $U_{\max }$, the friction coefficient $C_{f}$, the cavity width in the streamwise direction $s$, the cavity depth $d$, the time-averaged mixing layer thickness $\delta$, the pore Reynolds number $R e_{p}$, the roughness Reynolds number $R e_{d}$, and the mixing layer Reynolds number $R e_{m l}$. Time-averaged values are computed in the quasi-steady time window.

\begin{tabular}{|c|c|c|c|c|c|c|c|c|c|c|c|c|c|c|}
\hline Experiment & $U_{h_{c}}(\mathrm{~m} / \mathrm{s})$ & $\rho_{h_{c}}\left(\mathrm{~kg} / \mathrm{m}^{3}\right)$ & $h_{c}(\mathrm{~mm})$ & $R e_{b}$ & $F r_{b}$ & $U^{*}(\mathrm{~m} / \mathrm{s})$ & $U_{\max }(\mathrm{m} / \mathrm{s})$ & $C_{f}$ & $\mathrm{~s}(\mathrm{~mm})$ & $\mathrm{d}(\mathrm{mm})$ & $\delta(\mathrm{mm})$ & $R e_{p}$ & $R e_{d}$ & $R e_{m l}$ \\
\hline D1006_Q07_P00 & 0.037 & 1005.3 & 51 & 1879 & 0.71 & 0.0025 & 0.056 & $9.1 * 10^{(-3)}$ & 0 & 20 & 29 & 0 & 0 & 824 \\
\hline D1006_Q07_P12 & 0.048 & 1005.2 & 47 & 2312 & 0.96 & 0.0038 & 0.068 & $12.3 * 10^{(-3)}$ & 3 & 20 & 29 & 8 & 66 & 1514 \\
\hline D1006_Q07_P20 & 0.047 & 1005.1 & 45 & 2175 & 0.97 & 0.0046 & 0.068 & $19.0 * 10^{(-3)}$ & 5 & 20 & 29 & 21 & 79 & 1553 \\
\hline D1006_Q07_P29 & 0.054 & 1005.0 & 47 & 2285 & 0.95 & 0.0040 & 0.066 & $14.0 * 10^{(-3)}$ & 8 & 20 & 33 & 28 & 69 & 1687 \\
\hline D1006_Q07_P42 & 0.049 & 1005.0 & 47 & 2332 & 1.01 & 0.0048 & 0.067 & $18.7 * 10^{(-3)}$ & 15 & 20 & 40 & 62 & 83 & 2062 \\
\hline D1006_Q07_P42F & 0.038 & 1005.3 & 55 & 2083 & 0.69 & 0.0040 & 0.053 & $22.3 * 10^{(-3)}$ & 15 & 20 & 45 & 52 & 69 & 1153 \\
\hline D1006_Q12_P00 & 0.053 & 1005.3 & 77 & 4181 & 0.82 & 0.0035 & 0.073 & $8.6 * 10^{(-3)}$ & 0 & 20 & 29 & 0 & 0 & 1682 \\
\hline D1006_Q12_P12 & 0.062 & 1005.2 & 70 & 4379 & 1.01 & 0.0043 & 0.081 & $9.8 * 10^{(-3)}$ & 3 & 20 & 44 & 9 & 74 & 2577 \\
\hline D1006_Q12_P20 & 0.066 & 1005.0 & 68 & 4526 & 1.1 & 0.0042 & 0.085 & $8.1 * 10^{(-3)}$ & 5 & 20 & 33 & 20 & 72 & 1484 \\
\hline D1006_Q12_P29 & 0.066 & 1005.1 & 68 & 4477 & 1.05 & 0.0047 & 0.085 & $10.9 * 10^{(-3)}$ & 8 & 20 & 37 & 33 & 81 & 2323 \\
\hline D1006_Q12_P42 & 0.066 & 1005.2 & 70 & 4394 & 1.06 & 0.0052 & 0.086 & $13.6 * 10^{(-3)}$ & 15 & 20 & 56 & 67 & 90 & 3408 \\
\hline D1006_Q12_P42F & 0.052 & 1005.2 & 82 & 4283 & 0.78 & 0.0044 & 0.072 & $14.4 * 10^{(-3)}$ & 15 & 20 & 44 & 57 & 76 & 1185 \\
\hline D1006_Q14_P00 & 0.057 & 1004.9 & 94 & 5478 & 0.83 & 0.0031 & 0.082 & $5.8 * 10^{(-3)}$ & 0 & 20 & 33 & 0 & 0 & 1399 \\
\hline$D 1006 \_Q 14 \_P 12$ & 0.060 & 1005.0 & 88 & 5378 & 0.89 & 0.0046 & 0.084 & $11.5 * 10^{(-3)}$ & 3 & 20 & 48 & 10 & 79 & 2621 \\
\hline D1006_Q14_P20 & 0.068 & 1005.0 & 78 & 5430 & 1.08 & 0.0049 & 0.093 & $10.0 * 10^{(-3)}$ & 5 & 20 & 41 & 23 & 84 & 1774 \\
\hline D1006_Q14_P29 & 0.070 & 1004.9 & 78 & 5604 & 1.11 & 0.0047 & 0.094 & $9.14 * 10^{(-3)}$ & 8 & 20 & 45 & 33 & 81 & 3156 \\
\hline D1006_Q14_P42 & 0.068 & 1004.9 & 83 & 5697 & 1.06 & 0.0049 & 0.094 & $10.1 * 10^{(-3)}$ & 15 & 20 & 44 & 63 & 84 & 2839 \\
\hline D1006_Q14_P42F & 0.059 & 1005.1 & 87 & 5244 & 0.87 & 0.0042 & 0.079 & $12.5 * 10^{(-3)}$ & 15 & 20 & 59 & 54 & 72 & 3118 \\
\hline D1006_Q14_P29F & 0.053 & 1005.2 & 88 & 4682 & 0.76 & 0.0052 & 0.072 & $15.3 * 10^{(-3)}$ & 8 & 20 & 52 & 37 & 90 & 2689 \\
\hline D1020_Q13_P00 & 0.082 & 1017.4 & 57 & 4702 & 0.82 & 0.0053 & 0.115 & $8.3 * 10^{(-3)}$ & 0 & 20 & 39 & 0 & 0 & 3188 \\
\hline$D 1020 \_Q 13 \_P 12$ & 0.085 & 1016.6 & 52 & 4500 & 0.92 & 0.0050 & 0.123 & $7.1 * 10^{(-3)}$ & 3 & 20 & 24 & 11 & 86 & 2332 \\
\hline D1020_Q13_P20 & 0.088 & 1017.2 & 48 & 4368 & 0.96 & 0.0061 & 0.130 & $9.6 * 10^{(-3)}$ & 5 & 20 & 44 & 29 & 105 & 4312 \\
\hline D1020_Q13_P29 & 0.085 & 1017.2 & 52 & 4441 & 0.93 & 0.0060 & 0.125 & $9.4 * 10^{(-3)}$ & 8 & 20 & 40 & 43 & 103 & 3876 \\
\hline D1020_Q13_P42 & 0.092 & 1017.0 & 53 & 4910 & 0.96 & 0.0072 & 0.125 & $12.4 * 10^{(-3)}$ & 15 & 20 & 53 & 94 & 124 & 5208 \\
\hline D1020_Q13_P42F & 0.073 & 1017.5 & 68 & 5073 & 0.66 & 0.0062 & 0.098 & $14.7 * 10^{(-3)}$ & 15 & 20 & 44 & 81 & 107 & 3531 \\
\hline
\end{tabular}

Finally, the mixing layer forming at the porous substrate interface can be characterized by the Reynolds number,

$$
R e_{m l}=\frac{\delta U_{m l}}{v},
$$

where $\delta$ is the mixing layer thickness and $U_{m l}$ is the mean velocity averaged across $\delta$. If $R e_{m l}$ is taken as an indicator of the coherence of the boundary layer forming at the porous substrate interface, it can be generally (with few exceptions) stated that the mixing layer turbulence increases with porosity, initial inflow excess density, and inflow velocity.

Conversely, experiments performed over substrates filled with denser fluid are characterized by lower $R e_{b}, R e_{m} l$, and $R e_{p}$ values with respect to experiments performed over the same substrates filled with ambient water. This indicates that buoyancy induced instabilities contribute to turbulence generation, as it will be later discussed in Sec. III D 3.

\section{B. Density and velocity profiles}

\section{Vertical distribution of density}

Figure 7 shows the profiles of excess density, space-averaged over the porous substrate reach, and time-averaged in the sinking, transition, and quasi-steady phase, normalized by the initial excess density $\Delta \rho_{I}$ (see Fig. S1 of the supplementary material for a figure analogous to Fig. 7 but where variables are normalized with depth-averaged values). A logarithmic scale is applied to the $y$ axis to enhance differences between profiles. The main feature distinguishing density profiles measured over porous and nonporous beds is a convexity in the near-bed region, consequence of the mixing occurring across the porous substrate interface. It can be noted that higher porosities are associated with higher convexity in the lower part of the profile (i.e., for $z / H<0.12$ ): dilution is larger for porosities $\phi=0.2, \phi=0.29$, and $\phi=0.42$. Conversely, porosity $\phi=0.12$ has an intermediate behavior being more similar to the profile obtained over the flat bed. 

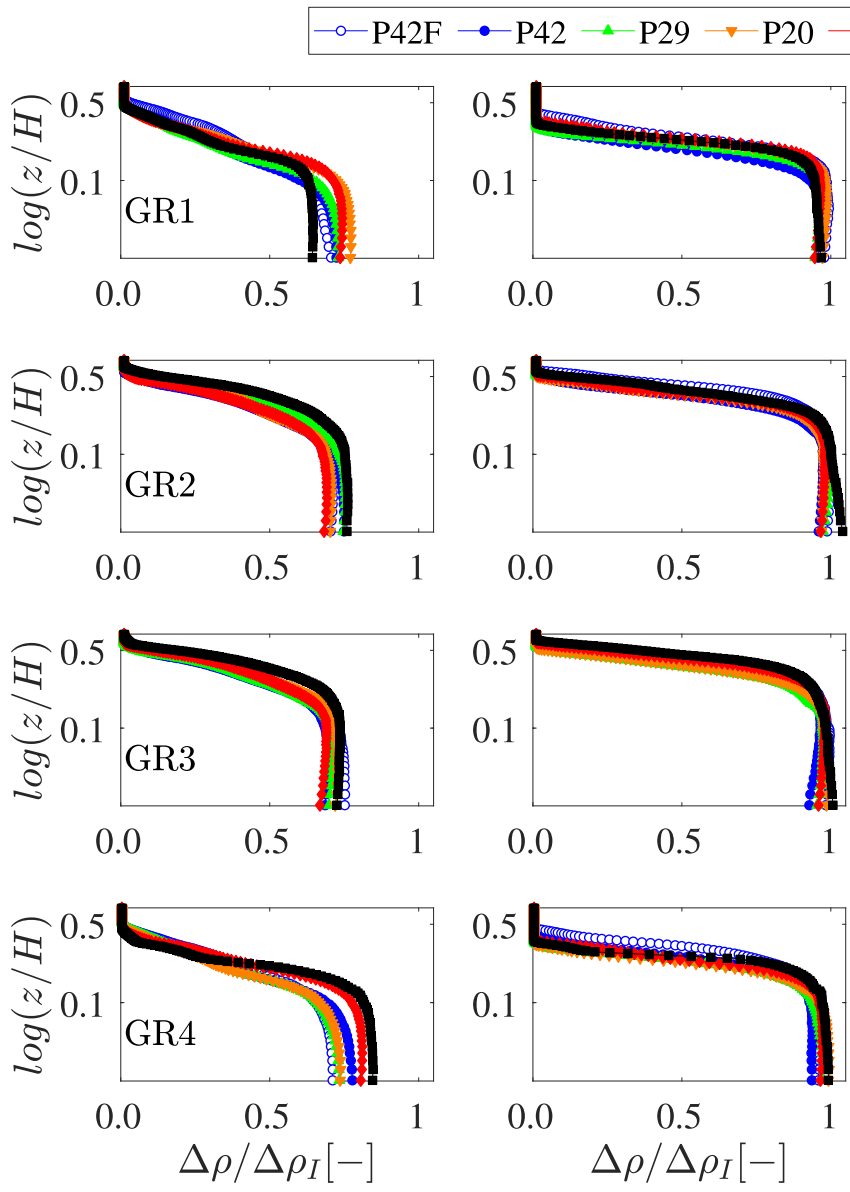

The convexity at the feet of time-averaged density profiles is observed during both, the transition and the steady state, with no remarkable difference.

\section{Vertical distribution of velocity}

In Fig. 8, normalized time-averaged velocities are represented as a function of the vertical coordinate $z$, normalized by the total depth $H$. The logarithmic scale for $z$ was chosen to enhance the profile differences in the near bed-region (see Fig. S3 of the supplementary material for a figure analogous to Fig. 8 but using a linear scale for the vertical axis). The inlet velocity $u_{I}$ is used to normalize velocity values (see Fig. S2 of the supplementary material for a figure analogous to Fig. 8 but where variables are normalized by depthaveraged values). What stands out at a first glance in Fig. 8 is that after the sinking phase, velocity profiles over higher porosities $(\phi=$ $0.2, \phi=0.29$, and $\phi=0.42$ ) clearly differentiate from velocity profiles acquired over filled or impermeable beds. Instead, if measured over low porosity substrates $(\phi=0.12)$, velocity profiles show intermediate behaviors (i.e., a behavior in between the nonporous case and cases with $\phi=0.42$ or $\phi=0.29$ ).

The main differences are observed in the region below the velocity maximum. Here, profiles measured over high porosity substrates are characterized by higher velocities with respect to the
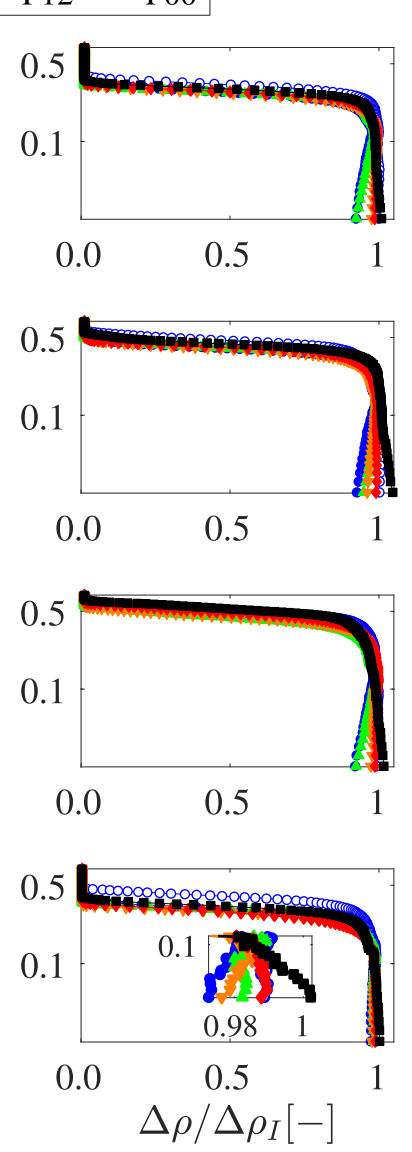

same experiments performed over low or null porosity substrates. The difference is especially evident in the near-bed region where, if the bed is permeable, velocity measurements are up to five times faster than over impermeable substrates. However, velocity values do not monotonically increase with substrate porosity: the largest velocity peak is observed for $\phi=0.2$ (orange in Fig. 8) for GR1 and GR4 experiments, while the velocity maximum is nearly the same for porosity $\phi=0.2, \phi=0.29$, and $\phi=0.42$ (see Table III) in GR2 and GR3 experiments. Moreover, looking at the region under the velocity maximum in GR2 experiments, the profile corresponding to $\phi=0.2$ (orange) shows larger velocities with respect to the one obtained for $\phi=0.42$ (blue) and $\phi=0.29$ (green). Analogously, in GR3 experiments, $\phi=0.29$ (green) shows larger velocities than $\phi=0.42$ (blue).

For GR1 and GR4 experiments, when the porous substrate is initially filled with denser fluid, velocities close to the porous substrate interface are larger than over impermeable beds but lower than over substrates initially saturated with freshwater.

As mentioned in Sec. III A 5, the existence of a longitudinal pressure gradient, consequence of the current interface slope, can explain the larger velocities observed over porous substrates. We argue that this velocity increase is not monotonic because two additional effects of the porous boundary intervene to shape the vertical 

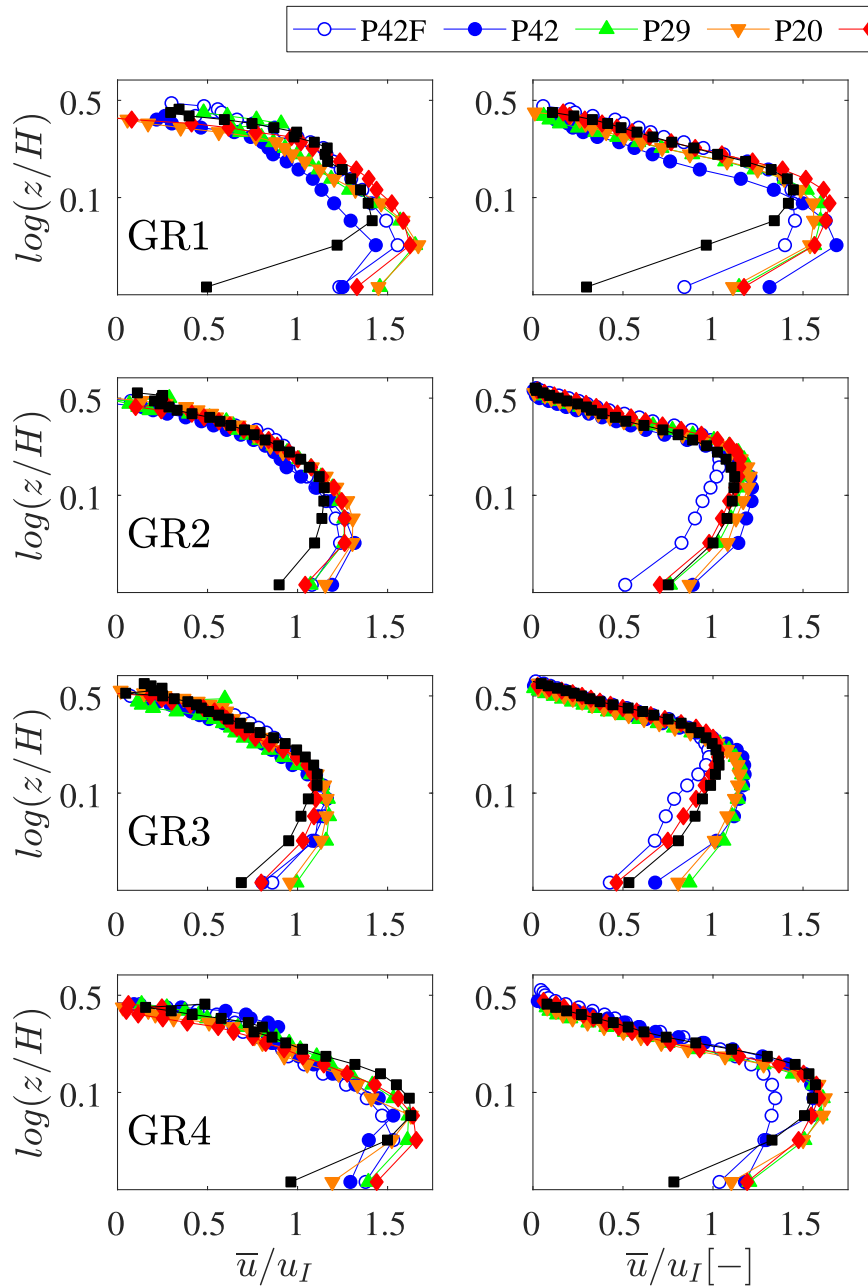

velocity profiles. These are the relaxation of the no-slip boundary condition and the bed-normal momentum exchange. They have opposite effects on the flow resistance and consequently on the velocity field: the former decreases the flow resistance allowing higher flow velocities close to the porous substrate interface and the latter enhances turbulent stresses, increasing the flow resistance and reducing the flow velocity.

While the velocity field in the near-bed region is significantly affected by the lower boundary condition, Fig. 8 shows that velocity profiles above the velocity maximum almost collapse. This indicates that in our experiments, the lower boundary conditions do not significantly affect the mixing and consequently the entrainment at the upper boundary of the current. On the contrary, the mean velocity gradient $d \bar{u} / d z$ computed in-between the velocity maximum and the velocity measure closest to the bed (we here omitted the figure for brevity) decreases over porous beds, becoming minimum for $\phi=0.2$ and $\phi=0.29$ and increasing again for higher porosities (although remaining lower than over solid beds). Values of $d \bar{u} / d z$ over porous but filled (with heavier fluid) substrates are lower than over nonporous beds, and comparable with values found over porous
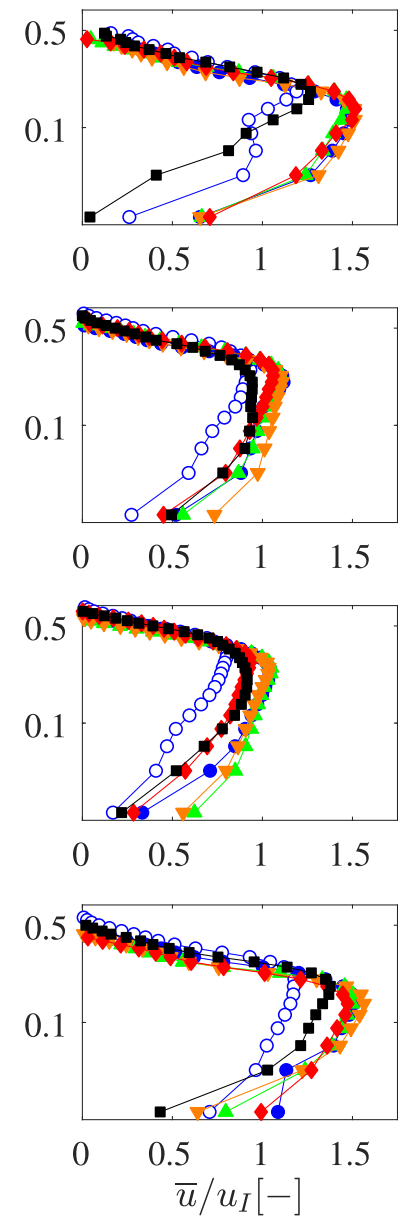

nonfilled beds. These observations are consistent with the findings of Breugem et al., ${ }^{25}$ who attributed the mean shear reduction over permeable beds to the relaxation of the no-slip boundary condition at the permeable bed surface.

Finally, it is interesting to note that the ratio between the front velocity and the depth-averaged body velocity (i.e., the depthaveraged velocity, time-averaged during the quasi-steady phase) decreases over larger porosity substrates. Particularly, it is slightly larger than one over large porosity substrates (i.e., $\phi \geq 0.2$ ), while over nonporous, low porosity $(\phi=0.12)$, or filled substrates, it is lower than one (see Fig. S4 of the supplementary material). Previous studies and field observations concerning gravity currents fed by continuous steady supply reported that the current body is faster than the current front. ${ }^{10,41}$ This is in disagreement with our experimental results over nonporous substrates but may be due to the different applied definition of current depth (resulting in different depth-averaged body velocities). Moreover, other field observations reported a faster front detaching from the body. This is typical of surge-like events ${ }^{42}$ but has also been observed in case of sustained events. ${ }^{43,44}$ 
D1006_Q14_P42_I
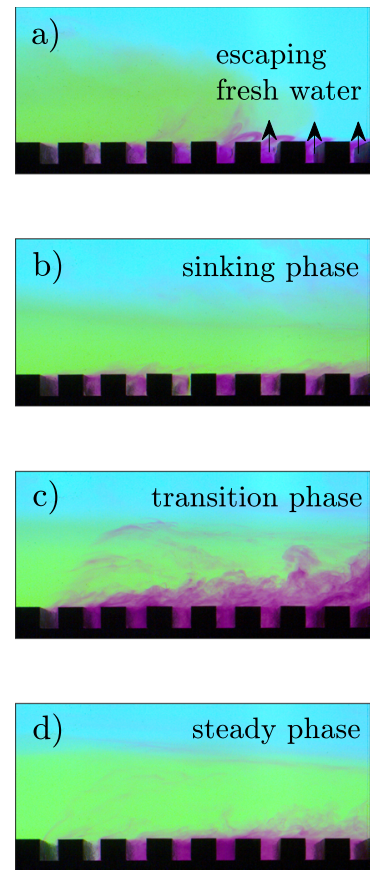

D1006_Q14_P42_IF
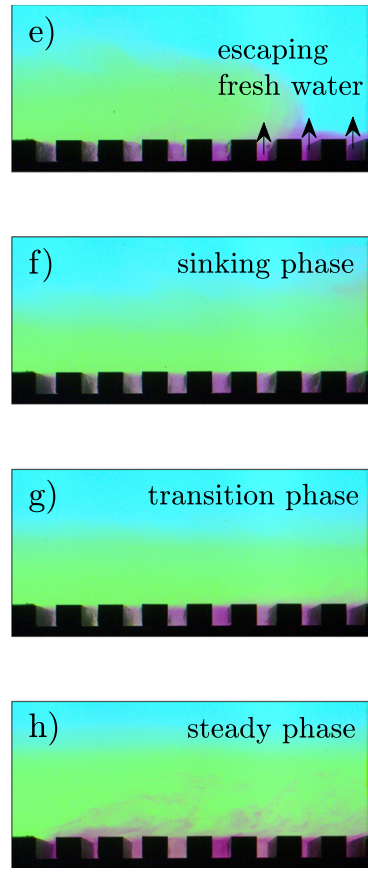

FIG. 9. Raw images acquired during two experiments having the same inflow conditions (D1006_Q14_P42) when the bed is filled with inked water of density $1000 \mathrm{~kg} / \mathrm{m}^{3}$ [in chronological order: (a)-(d)] and when the bed is filled with inked water of density $1006 \mathrm{~kg} / \mathrm{m}^{3}$ [in chronological order: (e)-(h)].

\section{Momentum exchange at the bed surface}

\section{Mixing layer at the bed surface}

Constant-density flows over permeable beds ${ }^{25-27}$ are characterized by the development of a turbulent boundary layer in the near-bed region. In our experiments, a mixing layer forms above the porous substrate interface (see Fig. 9). Its dynamics are driven by two types of instabilities occurring simultaneously: RayleighTaylor instabilities (buoyancy driven instabilities due to the presence of heavier fluid over lighter one) and shear-driven instabilities (shear induced instabilities due to the sharp velocity gradient at the porous substrate interface). Figure 9 shows the mixing layer time evolution in two experiments having the same inflow conditions (D1006_Q14_P42I) but performed over a bed filled with fresh inked water and over a bed filled with inked brine water (i.e., of the same density of the injected brine water), respectively. The outstanding difference in thickness and coherence of the mixing layer shows the importance of Rayleigh-Taylor instabilities in the mixing process at the lower boundary of the current. The local value of the gradient Richardson number quantifies the ratio between the static stability $-g / \bar{\rho} \partial \rho / \partial z$ and the dynamic instability due to the velocity gradient $\partial u / \partial z$

$$
R i(z)=-\frac{g}{\bar{\rho}} \frac{\partial \rho / \partial z}{(\partial u / \partial z)^{2}} .
$$

Figure 10 shows the time evolution of the gradient Richardson number at the penultimate available measurement point over the bed (i.e., about $7 \mathrm{~mm}$ above the porous substrate interface) and the time-averaged gradient Richardson number profile for the GR2 dataset.

The first remarkable observation concerns the sign of $\operatorname{Ri}(z)$ in the near bed region. Here, if the bed is porous, after the sinking phase, the gradient Richardson number becomes negative. This implies that the term $\partial \rho / \partial z$ is positive and therefore the premise for the development of static instabilities exists. Low porosities (i.e., $\phi=0.12$ ) and filled cases are characterized by smaller magnitudes but still negative values of Ri. On the contrary, when the bed is impervious, the gradient Richardson number remains positive although lower than the value 0.25 , considered the threshold over which the flow remains stable even in the presence of small shear perturbations. ${ }^{45}$ Indeed, the velocity gradient close to the bed is large, while the density gradient is small. We remark that the term $\partial \rho / \partial z$ is the density gradient above the bed; therefore, when positive, it indicates that lighter fluid has entered the flow over the bed surface or, in other words, it has been entrained into the current. Interestingly, during the sinking phase, no lighter fluid is entrained into the current and the gradient Richardson number is positive also for porous beds: no mixing layer is observed in this phase [see Figs. 9(b) and $9(\mathrm{f})]$. In the transition phase, instead, if the bed is porous, $\operatorname{Ri}(z)$ becomes negative, proving the mixing layer development and the consequent entrainment of bed freshwater. During this phase, Rayleigh-Taylor instabilities prevail over shear-driven instabilities
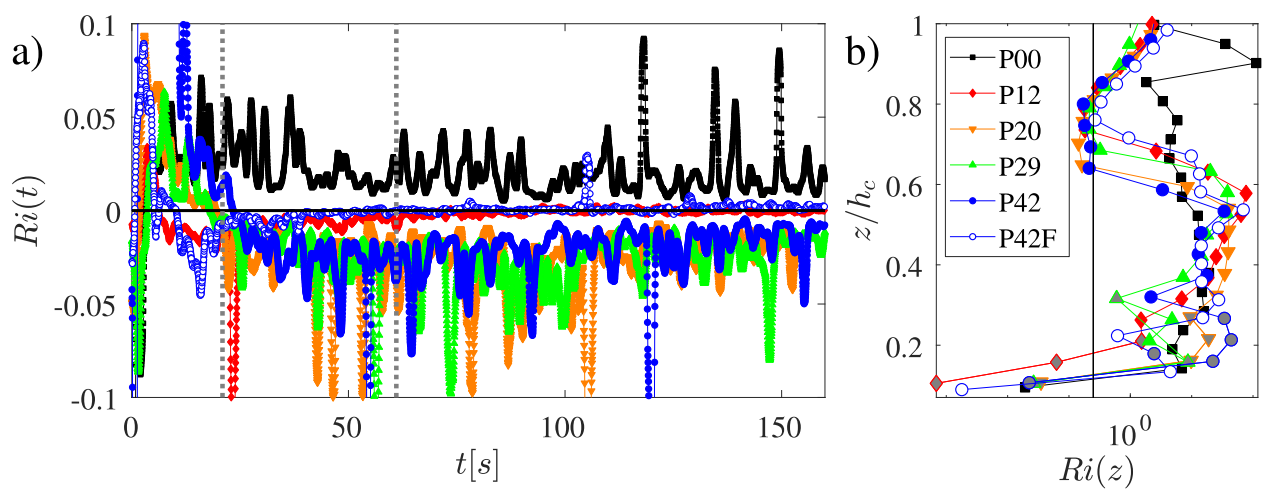

FIG. 10. (a) Time evolution of $R i$ at the penultimate available measurement point over the bed (i.e., about $7 \mathrm{~mm}$ above the bed). Dashed gray vertical lines identify the beginning of the transition and steady phases for the experiment P42. (b) Gradient Richardson number profiles time-averaged during the quasi-steady period. Data shown belong to $G R 2$ experiments. The horizontal axis scale is logarithmic. Negative Ri values are represented by markers filled in gray. The black continuous line indicates $R i=0.25$. 
and the mixing layer reaches its maximum thickness and coherence [see Fig. 9(c)]. On the other hand, if the surface flow has the same density of the fluid in the porous substrate, no static instabilities can develop, and the mixing layer does not form [see Fig. $9(\mathrm{~g})$ ].

During the steady state, as the porous substrate fills with denser fluid, the density difference between the surface and subsurface flow decreases. As a consequence, static instabilities progressively loose strength and the mixing layer thickness slowly shrinks [see Fig. 9(d)]. However, gradient Richardson values close to the bed remain negative for the entire experiment duration, proving that static instabilities never completely fade. During this phase the mixing layer dynamics are ruled by shear-driven instabilities. Therefore, mixing is observed even if no density difference exists between the surface and subsurface flows [see Fig. 9(h)].

Given the unsteadiness of the mixing layer forming at the porous substrate interface, to characterize its thickness, $\delta$, we chose the steady period as a time averaging window. We assumed the extension of the mixing layer must fall in-between the first (when moving from the porous substrate interface toward the ambient water surface) zero-crossing of Reynolds shear stress profiles [see Fig. 11(a)] and the zero-crossing of the time-averaged gradient Richardson profiles. For experiments carried out over impermeable boundaries, the zero-crossing of Reynolds shear stresses was used to determine the extension of the boundary layer. Table III lists obtained values of mixing layer thicknesses: $\delta$ is generally increasing with substrate porosity and inflow velocity. Conversely, the initial excess density has no relevant effect on $\delta$ values.

\section{Shear stresses and bed friction}

We evaluated the friction velocity, starting from its definition,

$$
U^{*}=\sqrt{\frac{\tau_{b e d}}{\rho}},
$$

where $\tau_{\text {bed }}$ is the shear stress at the bed level $(z=0)$. Note that $\tau_{\text {bed }}$ incorporates the effects of both the skin friction at the top of the PVC bars and the turbulent momentum flux through them. The total shear stress, $\tau(z)$, vertical profile reads

$$
\tau(z)=\mu \frac{d \bar{u}}{d z}-\rho \overline{u^{\prime} w^{\prime}}
$$

and can be observed for group 3 experiments in Fig. 11(c). Figures 11(a) and 11(b) show $\tau$ turbulent and viscous shear stress vertical distribution, respectively. In the near bed region, the order of magnitude of the two components is comparable. Over the impermeable bed, the term $\mu d u / d z$ is remarkably larger than the term $-\rho \overline{u^{\prime} w^{\prime}}$ meaning that viscous shear dominates. Over porous substrates, the importance of Reynolds shear stresses (especially in the lower half of the current depth) increases with increasing substrate porosity. We argue that this is a consequence of both the roughness of the porous boundary and the momentum exchange through it. The effect of the latter can be observed by comparing [in Fig. 11(a)] P42 and P42F experiments: $-\rho \overline{u^{\prime} w^{\prime}}$ are larger when the bed is filled with ambient water, proving that buoyancy induced instabilities enhance the momentum exchange through the porous interface. Comparing P00 and P42F experiments in the near bed region of Fig. 11(b), the effect of the relaxation of the no-slip boundary condition can be observed: the viscous shear is lower for P42F than for P00 experiment. Higher up, between $0.1<z / h_{c}<0.22$, the two are comparable, but, in general, lower viscous shear is observed over larger porosity substrates. This trend reverses in the region $0.22<z / h_{c}$ $<0.6$ and reverses again above $z=0.6 z / h_{c}$.

The lowest points of the total shear profiles [Fig. 11(c)] are linearly extrapolated [see Fig. 11(c)] to find their intersection with $z=0$, which is taken as estimation of bed shear stress $\tau_{\text {bed }}$. The obtained $U^{*}$ values are close to one tenth of the maximum of the velocity profile, which is an expected order of magnitude.

In Fig. 12(a), we compare the values of friction velocity obtained for the four groups of experiments. In general, the friction velocity increases monotonically with substrate porosity, regardless of the inflow conditions. Moreover, if experiments performed over substrates filled with denser fluid are compared with experiments performed over substrates filled with ambient water, the former show lower values of friction velocities with respect to the latter. This
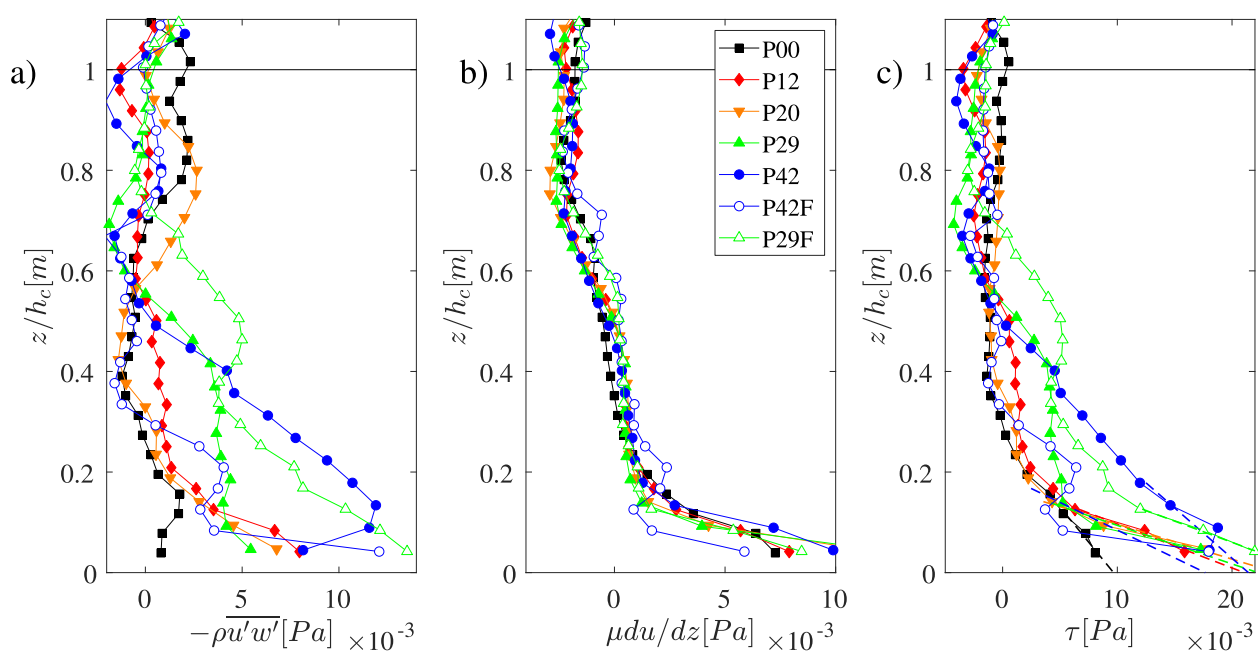

FIG. 11. (a) Turbulent shear stress profiles $\left(-\rho \overline{u^{\prime} w^{\prime}}\right)$. (b) Viscous shear stress profiles $(\mu d \bar{u} / d z)$. (c) Vertical distribution of the total shear $\tau$ and interpolation of the last points of the profile (dotted lines). Data shown refer to the steady state of GR3 experiments. The black horizontal line shows the position of the current interface. 


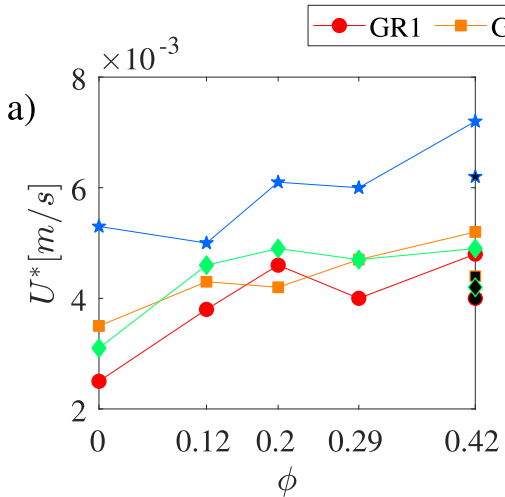

$-\mathrm{GR} 3 \rightarrow \mathrm{GR} 4$

observation stresses the effect of buoyancy induced instabilities on the flow resistance.

The obtained $U^{*}$ values are used to compute the friction coefficient, $C_{f}$, as ${ }^{25,46}$

$$
C_{f}=\frac{\tau_{b e d}}{0.5 \rho U_{h_{c}}^{2}}=2\left(\frac{U^{*}}{U_{h_{c}}}\right)^{2},
$$

where $U_{h_{c}}$ is the time-averaged depth-averaged velocity and $U^{*}$ is the friction velocity. The resulting $C_{f}$ values are listed in Table III and shown in Fig. 12(b). Consistently with previous finding, ${ }^{25}$ the skin friction coefficient increases with substrate porosity. It has been shown ${ }^{25,47}$ that when the flow regime is turbulent, skin friction increases over permeable beds. Conversely, when the flow is laminar, the relaxation of the no-slip-boundary condition at the porous substrate interface results in a decrease in skin friction. $^{48,49}$
Table III shows that in most of our experiments, the flow regime can be defined turbulent with few exceptions (mainly GR1 experiments). Therefore, our results confirm previous finding relative to constant-density flows over porous beds.

\section{Turbulence structure}

\section{Momentum fluxes and turbulent kinetic energy}

Figure 13 shows the Reynolds stress and turbulent kinetic energy (TKE) profiles, normalized by the square of the shear velocity, for varying porosities (data shown belong to group 3 experiments).

The presence of the porous substrate significantly affects the normal and shear Reynolds stress distribution. The main differences in streamwise turbulence $\overline{u^{\prime} u^{\prime}}$ profiles are observed in the near-bed region (i.e., for $z / h_{c}<0.2$ ). Here, larger peaks are observed over
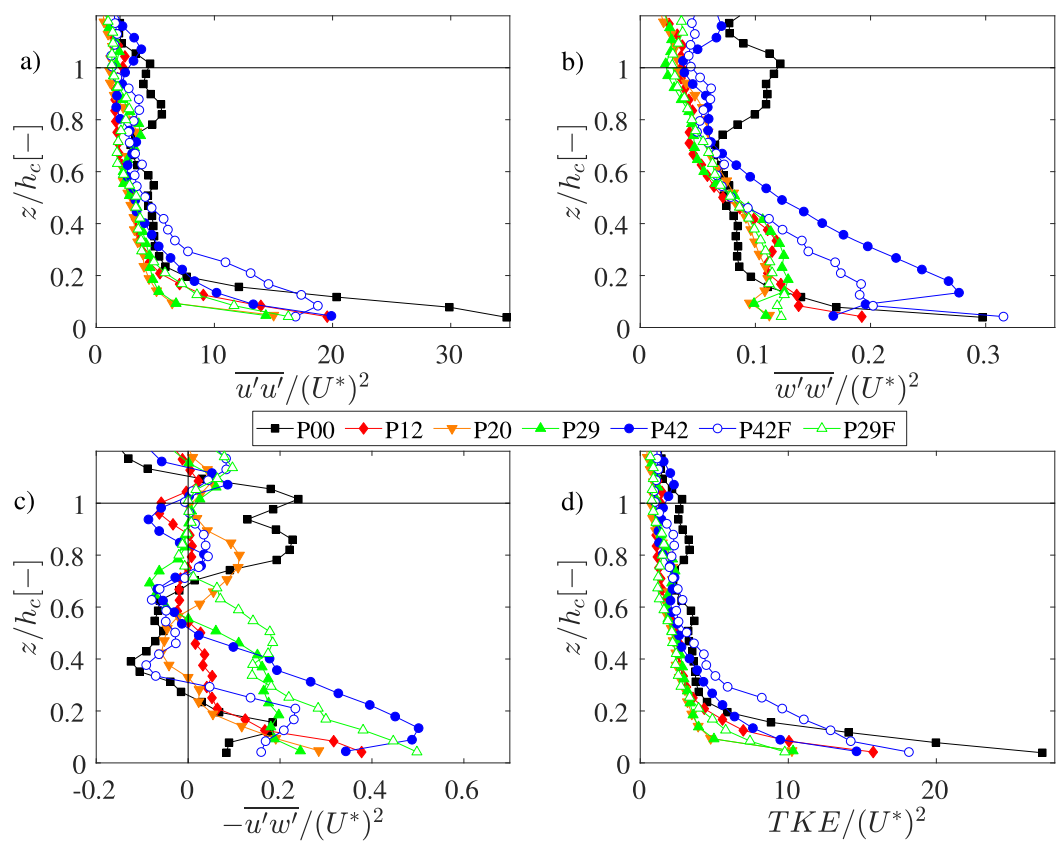

FIG. 13. Reynolds stress components $\overline{u^{\prime} u^{\prime}}(\mathrm{a}), \overline{w^{\prime} w^{\prime}}$ (b), $-\overline{u^{\prime} w^{\prime}}(\mathrm{c})$, and TKE (d) vertical distribution normalized by $U^{*^{2}}$. Data shown correspond to GR3 experiments. The black horizontal line identifies the location of the current interface. 
impermeable beds (compare P00, shown in black in Fig. 13, with remaining colorful profiles) and peak values are seen to decrease with increasing substrate porosity (except for the P42 experiment, shown with blue filled markers). This observation is in line to what has been previously observed by Breugem et al. ${ }^{25}$ and Manes et $a$ al $^{26,27}$ for constant density flows over permeable beds. They attributed the decrease in streamwise rms velocity to the lack of longitudinal low-high speed streaks and associated quasistreamwise vortices typical of solid beds. These longitudinal structures are destroyed by the turbulent transport in the bed-normal direction. Moreover, their formation requires strong shear regions, condition which fails over porous beds because of the relaxation of the no-slip boundary condition. ${ }^{2}$

As opposed to streamwise turbulence, the bed-normal turbulence, $\overline{w^{\prime} w^{\prime}}$, increases significantly over porous beds: the increment of $\overline{w^{\prime} w^{\prime}}$ stresses can be observed along almost the entire current body [i.e., up to $0.6 z / h_{c}$, see the P42 experiment shown in blue in Fig. 13(b)]. This is due to the absence of the wall blocking effect and, last but not least, to buoyancy. Indeed, the exchange between surface and subsurface flow is enhanced by static instabilities, as it can be noted comparing $\overline{u^{\prime} w^{\prime}}$ and $\overline{w^{\prime} w^{\prime}}$ profiles over porous ( $\mathrm{P} 42$, shown with blue filled markers) and porous but filled beds (P42F, represented with blue empty markers) in Figs. 13(b) and 13(c). Buoyancy effects also explain why larger vertical velocity fluctuations are observed far away above the porous substrate, which is not the case for constant density flow over porous beds.

Figure 13(d) shows the TKE vertical profile normalized with the square of the shear velocity, where TKE is computed as

$$
T K E=\frac{1}{2}\left(\overline{u^{\prime 2}}+\overline{v^{\prime 2}}+\overline{w^{\prime 2}}\right) .
$$

Despite the increase in bed-normal turbulence $\overline{w^{\prime 2}}$ over larger porosities, the decrease in streamwise turbulence $\overline{u^{\prime 2}}$ (which is the more significant contribution of turbulent kinetic energy) results in lower turbulent kinetic energy peaks above higher porosity substrates. This may sound counterintuitive considering that the shear velocity increase monotonically with the substrate porosity. On the other hand, this is observed because the turbulent shear stress and hence also the shear velocity depends on the correlation of the streamwise and normal velocity fluctuations (i.e., $-\overline{u^{\prime} w^{\prime}}$ ), while the turbulent kinetic energy depends on the normal stresses (i.e., $\overline{u^{\prime} u^{\prime}}$ and $\overline{w^{\prime} w^{\prime}}$ ) only.

Analogously to what observed by Tokyay et al., ${ }^{50}$ who investigated lock-exchange gravity currents propagating over an array of obstacles, the turbulent kinetic energy (TKE) profile has two peaks. The largest is found above the porous substrate interface, in the near bed region, while a smaller one appears close to the upper current interface (black line in Fig. 13), evidence of the presence of a shear layer. This relative peak is especially evident in TKE profiles of group 4 data, here not shown for brevity. Interestingly, its location moves closer to the bed for higher porosities. In accordance with what was observed by Kneller et al., ${ }^{51}$ Buckee et al. ${ }^{52}$, and Tokyay et al., ${ }^{50}$ the minimum of TKE is found approximately where the longitudinal velocity maximum and the minimum shear are observed (see Fig. S5 of the supplementary material). This is probably due to the fact that at this location, shear production of turbulence is almost null $(d u / d z=0)$ or to the fact that stratification may, at this height, be sufficiently strong to dissipate turbulence. ${ }^{52}$

Almost exactly where the TKE relative peak is found, a corresponding relative peak can be observed for Reynolds shear stresses, meaning that TKE production at the upper interface of the current is mainly due to mechanical shear (as buoyancy conditions are stable here). The largest peaks of Reynolds shear stresses are observed in the near-bed region above the porous substrate interface. Here, higher peaks are observed for larger porosities, although the observed trend is not monotonic: i.e., P29 (green) shows a smaller peak than P12 (red) and P20 (orange). Moreover, filled experiments (P42F, blue white filled markers, and P29F, green white filled markers) show different trends: the first indicating lower momentum exchange than its corresponding experiment P42 (thus proving buoyancy contribution to the enhancement of Reynolds shear stresses), the second indicating lower momentum exchange than its corresponding experiments P29 (which we attribute to high noise in the velocity measurements of this experiment).

The observed enhancement of Reynolds shear stresses is consistent with the observed increase in skin friction (see Sec. III C 2 or Table III).

Previous observations about the damping of streamwise turbulence and about the amplification of bed-normal turbulence over porous beds suggest a possible change in turbulence state when the substrate porosity changes. Indeed, turbulence is expected to approach a more three-dimensional isotropic state over permeable beds with respect to impermeable ones. To verify this hypothesis, we applied the so-called Lumley triangle technique ${ }^{53,54}$ in order to classify the turbulence state in the near-bed region. Figure 14 shows the turbulence state for the four velocity measurements closest to the bed of the four groups of experiments, when the substrate porosity varies. A modest indication of change in turbulence state can be observed. Particularly, over impervious beds, turbulence is characterized by cigar-shaped axisymmetric structures (as expected for flows characterized by low-high speed streaks and associated quasistreamwise vortices). Conversely, over higher porosities substrates,

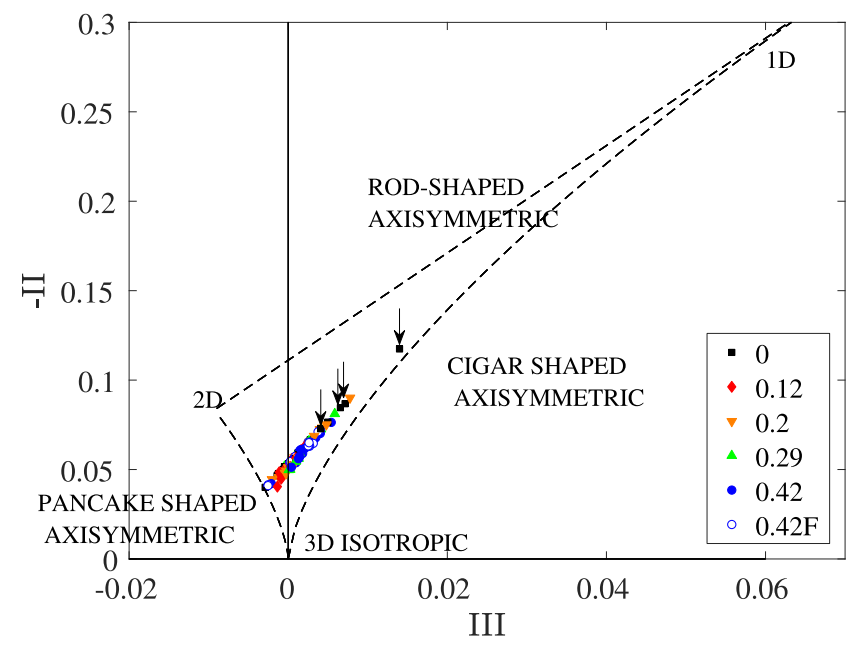

FIG. 14. Lumley triangle for the four groups of experiments (only the four points closest to the bed are shown), the arrows point the cases when $\phi=0$. 
it becomes more three-dimensional because bed-normal velocity fluctuations gain importance with respect to longitudinal ones, as previously observed by Breugem et al. ${ }^{25}$ among others.

\section{Mixing and buoyancy transport}

Figure 15 shows normalized time-averaged density fluctuation profiles $\overline{\rho^{\prime 2}}$ and buoyancy flux profiles $\overline{u^{\prime} \rho^{\prime}}, \overline{w^{\prime} \rho^{\prime}}$ for varying porosities. Data shown belong to group 3 experiments, and the chosen time averaging window is the steady phase. Normalization is made using the square of the initial excess density, $\rho_{I}$, and $U^{*} \rho_{I}$.

The largest peak in time-averaged density fluctuations profiles $\overline{\rho^{\prime 2}}$ is observed right below the upper current interface, where a shear induced mixing layer forms. Higher substrate porosities are associated with smaller peaks but thicker mixing layers. This result is a probably consequence of the current depth variation in time, which is related to the initial sinking, and therefore, it is stronger over porous beds.

In the near bed region [inset in Fig. 15(a)], $\overline{\rho^{\prime 2}}$, profiles show a concavity, evidence of the mixing occurring at the porous substrate interface. The higher the substrate porosity, the larger $\overline{\rho^{\prime 2}}$ the mixing. Consistently, experiments performed over filled or solid beds do not show the mentioned concavity.

Time-averaged profiles of the buoyancy flux $\overline{u^{\prime} \rho^{\prime}}$ are quite similar to $\overline{\rho^{\prime 2}}$ profiles, peaking in the mixing layer right below the upper current interface. Interestingly, experiments carried out over solid or filled bottoms show larger peaks, suggesting mixing at the upper interface is stronger when no sinking occurs. $\overline{u^{\prime} \rho^{\prime}}$ values increase progressively in the near bed region, reaching a relative maximum close to the bed [see the inset in Fig. 15(b)] where larger mixing occurs.

At the same $z$ location where $\overline{\rho^{\prime 2}}$ and $\overline{u^{\prime} \rho^{\prime}}$ peak, in the upper shear layer of the current, a negative peak in $\overline{w^{\prime} \rho^{\prime}}$ profiles can be found. In general, $\overline{w^{\prime} \rho^{\prime}}$ becomes negative in the upper half of the current, while in the lower half, especially for larger porosities, it is positive (except for $\phi=0.2$ data, which are in disagreement with the general trend). For $\phi=0.42$ and $\phi=0.29$, it can be observed that $\overline{w^{\prime} \rho^{\prime}}$ profiles remain positive for large portion of the current depth: up to $z / h_{c}=0.8$. This suggests that buoyancy effects are not limited to the near-bed region but extend upwards, as confirmed by normalized $\overline{w^{\prime} w^{\prime}}$ profiles in Fig. 13.

\section{Turbulent kinetic energy generation and dissipation}

The TKE budget equation reads ${ }^{55}$

$$
\frac{\partial T K E}{\partial t}=\frac{g \overline{w^{\prime} \rho^{\prime}}}{\rho}-\overline{u^{\prime} w^{\prime}} \frac{\partial u}{\partial z}-\frac{\partial \overline{w^{\prime} T K E}}{\partial z}-\frac{1}{\rho} \frac{\partial \overline{w^{\prime} p^{\prime}}}{\partial z}-\epsilon .
$$

The first two terms of the rhs of Eq. (15) represent source/sink terms that quantify the respective contributions to the TKE budget by buoyancy and mechanical shear. ${ }^{52}$ In statically unstable conditions (i.e., when lighter fluid lies below heavier fluid), buoyancy contributes to TKE generation and the term $g \overline{w^{\prime} \rho^{\prime}} / \rho$ is positive. On the contrary, in statically stable conditions (i.e., when lighter fluid lies over heavier fluid), buoyancy consumes TKE and the term $g \overline{w^{\prime} \rho^{\prime}} / \rho$ is negative. Similarly, negative values of $-\overline{u^{\prime} w^{\prime}} d \bar{u} / d z$ indicate that shear dissipates TKE and positive values of $-\overline{u^{\prime} w^{\prime}} d \bar{u} / d z$ indicate that shear produces TKE.

Figure 16 compares the relative contribution of buoyancy and shear in the TKE budget [Eq. (15)] for GR3 experiments.

In the near-bed region, if the substrate porosity is large (i.e., $\phi=0.29$ or $\phi=0.42$ ), both mechanical shear and buoyancy contribute to TKE generation. Indeed, for larger substrate porosities, up to $z / h_{c}=0.4$, both $-\overline{u^{\prime} w^{\prime}} d \bar{u} / d z$ and $g^{\prime} / \rho \overline{w^{\prime} \rho^{\prime}}$ are positive. However, buoyancy contribution is here $\left(z / h_{c}<0.4\right)$, one order of magnitude lower than the mechanical shear contribution. For some experiments, buoyancy production of TKE concerns a large part of the current depth: up to $z / h_{c}=0.7$ for $\phi=0.42$ and up to $z / h_{c}=1$ for $\phi=0.29$. Conversely, for experiments carried out over impermeable or low porosity substrates, TKE generation in the near-bed region is mainly due to mechanical shear: $g \overline{w^{\prime} \rho^{\prime}} / \rho$ is null or slightly negative for $\phi=0$ or $\phi=0.12$.

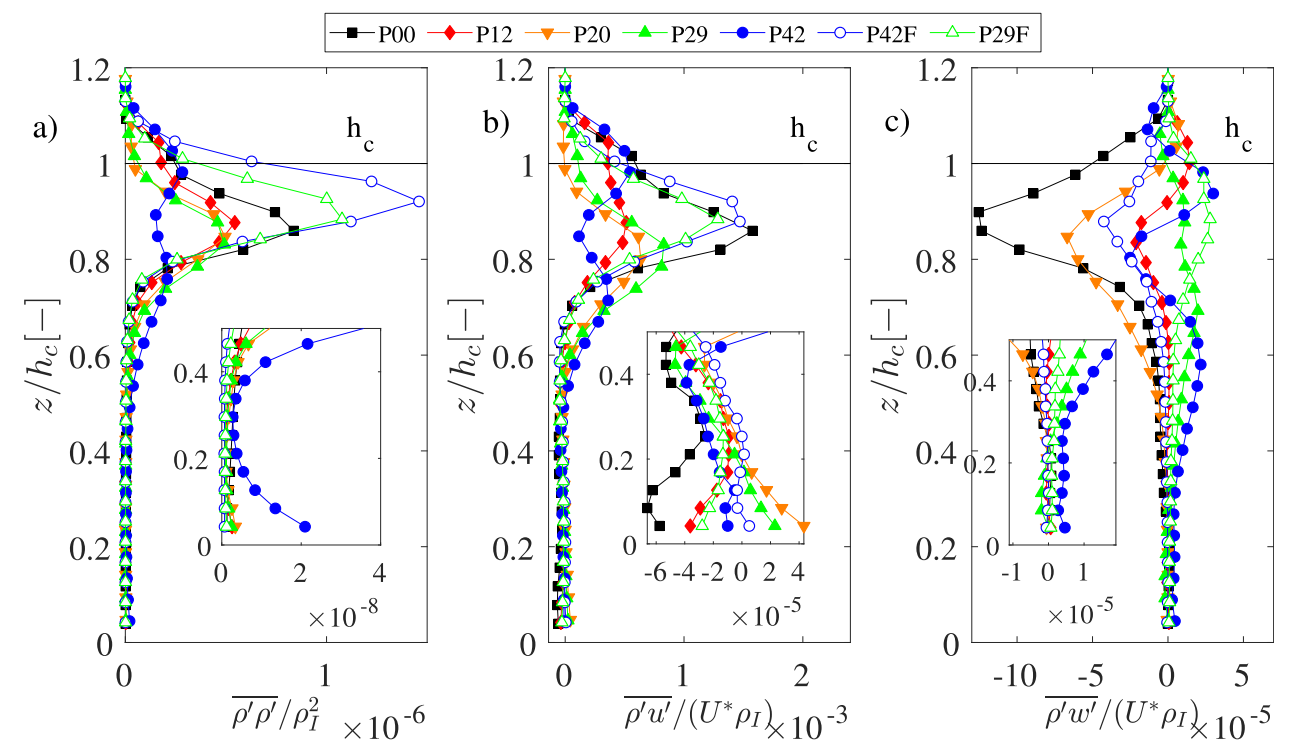

FIG. 15. (a) Time-averaged profiles of $\overline{\rho^{\prime 2}} /\left(\rho_{I}\right)^{2}$. (b) Time-averaged profiles of the normalized buoyancy flux $\overline{u^{\prime} \rho^{\prime}} /\left(U^{*} \rho_{I}\right)$. (c) Time-averaged profiles of the normalized buoyancy flux $\overline{w^{\prime} \rho^{\prime}}$. The black, horizontal line shows the location of the current interface. The chosen time averaging window is the steady phase. Data shown belong to the GR3 dataset. 

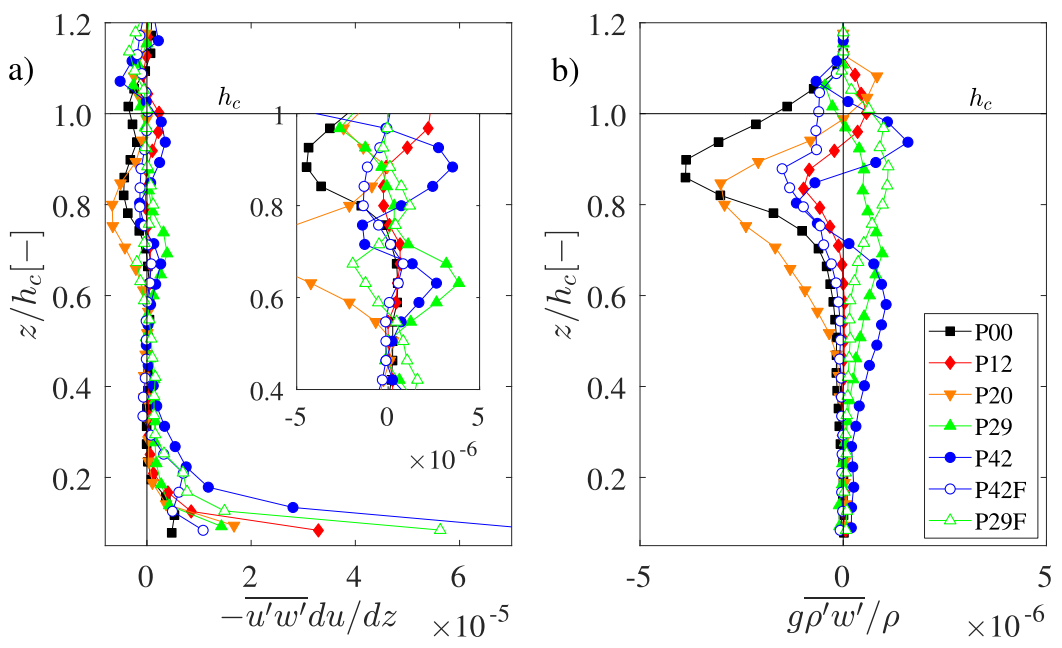

FIG. 16. Contribution to the TKE budget by mechanical shear (a) and buoyancy (b). Data shown concern GR3 experiments. Negative values of $-\overline{u^{\prime} w^{\prime}} d \bar{u} / d z$ and $g \overline{w^{\prime} \rho^{\prime}} / \rho$ indicate TKE consumption, while positive values of $-\overline{u^{\prime} w^{\prime}} d \bar{u} / d z$ and $g \overline{w^{\prime} \rho^{\prime}} / \rho$ indicate TKE generation.
Between $z / h_{c}=0.4$ and $z / h_{c}=1$, in the upper half of the current depth, the order of magnitude of shear and buoyancy contributions are comparable [see the inset in Fig. 16(a)]. A negative peak in $g \overline{w^{\prime} \rho^{\prime}} / \rho$ vertical distribution is observed in the region of the upper mixing layer, below the upper current interface (around at $\left.z / h_{c}=0.8\right)$. Here, buoyancy tends to dissipate turbulence: statically stable conditions are attained and shear induced disturbances are small (when positive $-\overline{u^{\prime} w^{\prime}} d \bar{u} / d z$ is comparable with the negative contribution of $g \overline{w^{\prime} \rho^{\prime}} / \rho$ ). Damping of turbulence due to buoyancy effects is larger over nonporous beds (i.e., larger negative peaks of $g \overline{w^{\prime} \rho^{\prime}} / \rho$ are observed for $\left.\phi=0\right)$. In fact, in these cases, at the upper current interface, stratification is stronger.

The role of mechanical shear at $z=h_{c}$ is not clear from Fig. 16(a). However, the general trend characterizing the four groups of experiments (see Fig. S6 of the supplementary material) indicates that $-\overline{u^{\prime} w^{\prime}} d \bar{u} / d z$, independently of the substrate porosity, generates TKE at the upper current interface. However, its contribution at $z=h_{c}$ is small when compared to the one at $z=0$.

\section{SUMMARY AND CONCLUSIONS}

This paper presents the results of 25 laboratory experiments involving continuously-fed brine density currents propagating first over an impermeable smooth bed and then over a porous substrate. Four different porosities of the underlying substrate were tested. We investigated the effects of the presence of the porous boundary on the velocity field, the density distribution, and the turbulent structure of density currents. Results show that when a gravity current overflows a porous substrate (even if of limited length), its mean flow and turbulent structure can change significantly. Our main observations are summarized hereafter:

- Three main flow phases are identified: a sinking phase, a transition phase, and finally a quasi-steady state. During the first, the current propagates sinking into the substrate, but no significant slowdown is remarked. At the beginning of the transition phase, freshwater entrainment from the bed starts and an unsteady mixing layer develops at the lower current boundary.
- Over porous beds, density current depths, $h_{c}$, are smaller with respect to those observed over impermeable beds or porous beds filled with denser fluid. However, $h_{c}$ does not decrease monotonically with increasing substrate porosity. This is due to the simultaneous occurrence of two opposite effects: the current sinking (resulting in lower $h_{c}$ ) and the larger bottom freshwater incorporation (feeding the current body and causing it to be thicker).

- The current interface over the porous substrate is generally characterized by a longitudinal slope (i.e., $h_{c}$ decreases in the flow direction). The larger the substrate porosity, the steeper the observed slope. Its occurrence, consequence of the current mass loss, results in the formation of a streamwise pressure gradient.

- The vertical distributions of mean flow variables (density and velocity) are affected by the presence of the porous boundary, especially in the near-bed region. Here, timeaveraged density profiles exhibit a marked concavity, the result of the dilution, and mixing occurring at the porous substrate interface.

- Velocity vertical distributions are mainly affected in the region below the velocity maximum. In this region, higher velocity values are recorded if the bed is porous, an effect of the streamwise pressure gradient. However, two other consequences of the presence of the porous substrate also affect the flow velocity distribution: the relaxation of the no-slip boundary condition at the porous substrate interface and bed the normal momentum exchange. Specifically, the former reduces the bulk flow resistance, while the latter increases it by enhancing turbulent shear stresses. We argue that the simultaneous occurrence of these opposite porous boundary effects results in the observed nonmonotonic velocity increase.

- The structure of the current above the porous bed is characterized by the presence of two mixing layers: developing at its upper and lower interfaces. The first is driven by shear induced instabilities and develops in statically stable conditions (i.e., buoyancy tends to damp shear induced instabilities); the second is driven by both Rayleigh-Taylor 
and shear-driven instabilities and develops in statically unstable conditions. While the mixing layer developing at the lower interface is significantly affected by the porosity of the underlying porous substrate, there was no significant effect of the substrate porosity on the velocity gradients and on the entrainment occurring at the upper current interface.

- The friction velocity $U^{*}=\sqrt{\tau_{\text {bed }} / \rho}$ (estimated extrapolating the vertical profiles of the total shear stress to the bed level to evaluate $\tau_{\text {bed }}$ ) increases with substrate porosity. This confirms previous findings, concerning constant density and turbulent flows over porous substrates. ${ }^{25-27}$

- The turbulent structure of the flow is significantly affected by the presence of the porous substrate: the momentum exchange through its interface enhances Reynolds stresses, especially in the bed-near region, where Reynolds shear stresses and wall-normal Reynolds stresses increase. On the contrary, the streamwise turbulence decreases due to the disappearance of low-high speed longitudinal streaks and associated quasistreamwise vortices, typically observed over solid beds and in regions of strong velocity shear (a condition which fails over the porous bed). Consequently, the largest peak of TKE vertical distribution, located over the porous substrate interface, decreases when the substrate porosity is larger (although not monotonically). These observations were already made for constant density flows over porous beds. ${ }^{25}$ However, in the present case, buoyancy further enhances the bednormal momentum flux and a larger portion of the flow depth is affected by the presence of the porous bed.

- In the near bed region, buoyancy contributes to TKE generation together with the mechanical shear $-\overline{u^{\prime} w^{\prime}} d \bar{u} / d z$. Conversely, at the upper current interface, buoyancy consumes TKE counteracting the mechanical shear generation effect.

\section{SUPPLEMENTARY MATERIAL}

See the supplementary material for Figs. S1-S6: Figure S1Fig. 7 where variables are normalized with depth-averaged values. Figure S2-Fig. 8 where variables are normalized with depthaveraged values. Figure S3-Fig. 8 where a linear scale is used for the vertical axis. Figure S4-Figure comparing the front velocity with the body depth-averaged velocity. Figure S5-Figure showing TKE profiles and time-averaged velocity profiles. Figure S6-Figure showing the contribution to the TKE budget by mechanical shear and buoyancy for GR1 experiment.

\section{ACKNOWLEDGMENTS}

The present work was funded by the Swiss National Science Foundation (SNSF, Grant No. 200021159249). The second author acknowledges support from The Binks Trust. The authors would like to acknowledge Dr. O. Sequeiros and the second anonymous reviewer for the constructive comments and suggestions which allowed us to significantly improve the earlier version of this article.

\section{REFERENCES}

${ }^{1}$ J. E. Simpson, Gravity Currents: In the Environment and the Laboratory (Cambridge University Press, 1997).

${ }^{2}$ M. La Rocca, C. Adduce, G. Sciortino, and A. B. Pinzon, "Experimental and numerical simulation of three-dimensional gravity currents on smooth and rough bottom," Phys. Fluids 20, 106603 (2008).

${ }^{3}$ T. M. Özgökmen and P. F. Fischer, "On the role of bottom roughness in overflows," Ocean Modell. 20, 336-361 (2008).

${ }^{4}$ T. Tokyay, G. Constantinescu, and E. Meiburg, "Lock-exchange gravity currents with a high volume of release propagating over a periodic array of obstacles," J. Fluid Mech. 672, 570-605 (2011).

${ }^{5} \mathrm{~T}$. Tokyay and G. Constantinescu, "The effects of a submerged non-erodible triangular obstacle on bottom propagating gravity currents," Phys. Fluids 27, 056601 (2015).

${ }^{6}$ W. D. Peters and J. E. S. Venart, "Rough-surface gravity current flows," Ph.D. thesis, The University of New Brunswick, Canada, 1999.

${ }^{7}$ Y. Tanino, H. M. Nepf, and P. S. Kulis, "Gravity currents in aquatic canopies," Water Resour. Res. 41, W12402 (2005).

${ }^{8}$ O. E. Sequeiros, B. Spinewine, R. T. Beaubouef, T. Sun, M. H. Garcia, and G. Parker, "Characteristics of velocity and excess density profiles of saline underflows and turbidity currents flowing over a mobile bed," J. Hydraul. Eng. 136, $412-433$ (2010).

${ }^{9}$ H. I. S. Nogueira, C. Adduce, E. Alves, and M. J. Franca, "Analysis of lockexchange gravity currents over smooth and rough beds," J. Hydraul. Res. 51, 417-431 (2013).

${ }^{10}$ M. Stagnaro and M. Bolla Pittaluga, "Velocity and concentration profiles of saline and turbidity currents flowing in a straight channel under quasi-uniform conditions," Earth Surf. Dyn. 2, 167-180 (2014).

${ }^{11}$ F. Y. Testik and N. A. Yilmaz, "Anatomy and propagation dynamics of continuous-flux release bottom gravity currents through emergent aquatic vegetation," Phys. Fluids 27, 056603 (2015).

${ }^{12}$ C. Cenedese, R. Nokes, and J. Hyatt, "Lock-exchange gravity currents over rough bottoms," Environ. Fluid Mech. 18, 59-73 (2018).

${ }^{13}$ E. B. Dussan V. and F. M. Auzerais, "Buoyancy-induced flow in porous media generated near a drilled oil well. Part 1 . The accumulation of filtrate at a horizontal impermeable boundary," J. Fluid Mech. 254, 283-311 (1993).

${ }^{14}$ R. L. Doneker, J. D. Nash, and G. H. Jirka, "Pollutant transport and mixing zone simulation of sediment density currents," J. Hydraul. Eng. 130, 349-359 (2004).

${ }^{15}$ L. P. Thomas and B. M. Marino, "Inertial density currents over porous media limited by different lower boundary conditions," J. Hydraul. Eng. 138, 133-142 (2012).

${ }^{16}$ J. Lionet and O. Quoy, "Gravity currents over porous media," Internal Report No. DAMTP, University of Cambridge, Cambridge, 1995.

${ }^{17}$ L. P. Thomas, B. M. Marino, and P. F. Linden, "Gravity currents over porous substrates,” J. Fluid Mech. 366, 239-258 (1998).

${ }^{18}$ B. M. Marino and L. P. Thomas, "Spreading of a gravity current over a permeable surface," J. Hydraul. Eng. 128, 527-533 (2002).

${ }^{19}$ T. B. Moodie and J. P. Pascal, "Downslope movement of compositionally driven gravity flows over porous surfaces,” J. Porous Media 2, 127-141 (1999).

${ }^{20}$ T. B. Moodie and J. P. Pascal, "Axisymmetric spreading and filtration of inclined thermals over porous surfaces," Can. Appl. Math. Q 7, 185-201 (1999).

${ }^{21} \mathrm{M}$. Ungarish and H. E. Huppert, "High-Reynolds-number gravity currents over a porous boundary: Shallow-water solutions and box-model approximations," J. Fluid Mech. 418, 1-23 (2000).

${ }^{22}$ J. M. Acton, H. E. Huppert, and M. G. Worster, "Two-dimensional viscous gravity currents flowing over a deep porous medium," J. Fluid Mech. 440, 359-380 (2001).

${ }^{23}$ L. P. Thomas, B. M. Marino, and P. F. Linden, "Lock-release inertial gravity currents over a thick porous layer,” J. Fluid Mech. 503, 299-319 (2004).

${ }^{24}$ B. Guo, Z. Zheng, M. A. Celia, and H. A. Stone, "Axisymmetric flows from fluid injection into a confined porous medium," Phys. Fluids 28, 022107 (2016).

${ }^{25}$ W. P. Breugem, B. J. Boersma, and R. E. Uittenbogaard, "The influence of wall permeability on turbulent channel flow," J. Fluid Mech. 562, 35-72 (2006). 
${ }^{26}$ C. Manes, D. Pokrajac, I. McEwan, and V. Nikora, "Turbulence structure of open channel flows over permeable and impermeable beds: A comparative study," Phys. Fluids 21, 125109 (2009).

${ }^{27}$ C. Manes, D. Poggi, and L. Ridolfi, "Turbulent boundary layers over permeable walls: Scaling and near-wall structure," J. Fluid Mech. 687, 141-170 (2011).

${ }^{28} \mathrm{M}$. Negretti, D. Z. Zhu, and G. H. Jirka, "The effect of bottom roughness in two-layer flows down a slope," Dyn. Atmos. Oceans 45, 46-68 (2008).

${ }^{29}$ O. E. Sequeiros, "Estimating turbidity current conditions from channel morphology: A Froude number approach," J. Geophys. Res.: Oceans 117, C04003, https://doi.org/10.1029/2011jc007201 (2012).

${ }^{30}$ H. I. S. Nogueira, C. Adduce, E. Alves, and M. J. Franca, "Image analysis technique applied to lock-exchange gravity currents," Meas. Sci. Technol. 24, 047001 (2013).

${ }^{31}$ S. Balasubramanian and Q. Zhong, "Entrainment and mixing in gravity currents using simultaneous velocity-density measurements," Phys. Fluids 30, 056601 (2018); e-print arXiv:1802.02364.

${ }^{32} \mathrm{~K}$. Blanckaert and U. Lemmin, "Means of noise reduction in acoustic turbulence measurements," J. Hydraul. Res. 44, 3-17 (2006).

${ }^{33} \mathrm{M}$. J. Franca and U. Lemmin, "Eliminating velocity aliasing in acoustic Doppler velocity profiler data," Meas. Sci. Technol. 17, 313-322 (2006).

${ }^{34}$ D. G. Goring and V. I. Nikora, "Despiking acoustic Doppler velocimeter data," J. Hydraul. Eng. 128, 117-126 (2002).

${ }^{35}$ L. Ottolenghi, C. Adduce, R. Inghilesi, F. Roman, and V. Armenio, "Mixing in lock-release gravity currents propagating up a slope," Phys. Fluids 28, 056604 (2016).

${ }^{36}$ R. E. Britter and P. F. Linden, "The motion of the front of a gravity current travelling down an incline," J. Fluid Mech. 99, 531-543 (1980).

${ }^{37}$ T. M. Özgökmen and E. P. Chassignet, "Dynamics of two-dimensional turbulent bottom gravity currents,” J. Phys. Oceanogr. 32, 1460-1478 (2002).

${ }^{38} \mathrm{~J}$. J. Monaghan, R. A. F. Cas, A. M. Kos, and M. Hallworth, "Gravity currents descending a ramp in a stratified tank," J. Fluid Mech. 379, 39-69 (1999).

${ }^{39}$ H. Huang, J. Imran, C. Pirmez, Q. Zhang, and G. Chen, "The critical densimetric Froude number of subaqueous gravity currents can be non-unity or non-existent," J. Sediment. Res. 79, 479-485 (2009).

${ }^{40} \mathrm{~S}$. Leonardi, P. Orlandi, and R. A. Antonia, "Properties of d- and k-type roughness in a turbulent channel flow," Phys. Fluids 19, 125101 (2007).

${ }^{41}$ O. E. Sequeiros, R. Mosquera, and F. Pedocchi, "Internal structure of a self-accelerating turbidity current," J. Geophys. Res.: Oceans 123, 6260-6276, https://doi.org/10.1029/2018jc014061 (2018).
${ }^{42}$ J. E. Hughes Clarke, "First wide-angle view of channelized turbidity currents links migrating cyclic steps to flow characteristics," Nat. Commun. 7, 11896 (2016).

${ }^{43}$ J. P. Xu, M. A. Noble, and L. K. Rosenfeld, "In-situ measurements of velocity structure within turbidity currents," Geophys. Res. Lett. 31, L09311, https://doi.org/10.1029/2004gl019718 (2004).

${ }^{44}$ M. Azpiroz-Zabala, M. J. B. Cartigny, P. J. Talling, D. R. Parsons, E. J. Sumner, M. A. Clare, S. M. Simmons, C. Cooper, and E. L. Pope, "Newly recognized turbidity current structure can explain prolonged flushing of submarine canyons," Sci. Adv. 3, e1700200 (2017).

${ }^{45}$ J. J. Rohr, E. C. Itsweire, K. N. Helland, and C. W. V. Atta, "Growth and decay of turbulence in a stably stratified shear flow," J. Fluid Mech. 195, 77 (1988).

${ }^{46}$ F. M. White, Fluid Mechanics (McGraw-Hill, New York, NY, 2011).

${ }^{47}$ F. Kong and J. Schetz, "Turbulent boundary layer over solid and porous surfaces with small roughness," in 19th Aerospace Sciences Meeting, Aerospace Sciences Meetings (American Institute of Aeronautics and Astronautics, 1981).

${ }^{48}$ G. S. Beavers and D. D. Joseph, "Boundary conditions at a naturally permeable wall," J. Fluid Mech. 30, 197-207 (1967).

${ }^{49}$ S. Hahn, J. Je, and H. Choi, "Direct numerical simulation of turbulent channel flow with permeable walls," J. Fluid Mech. 450, 259-285 (2002).

${ }^{50}$ T. Tokyay, G. Constantinescu, and E. Meiburg, "Tail structure and bed friction velocity distribution of gravity currents propagating over an array of obstacles," J. Fluid Mech. 694, 252-291 (2012).

${ }^{51}$ B. C. Kneller, S. J. Bennett, and W. D. McCaffrey, "Velocity structure, turbulence and fluid stresses in experimental gravity currents," J. Geophys. Res.: Oceans 104, 5381-5391, https://doi.org/10.1029/1998jc900077 (1999).

${ }^{52}$ C. Buckee, B. Kneller, and J. Peakall, "Turbulence structure in steady, solutedriven gravity currents," in Particulate Gravity Currents (John Wiley and Sons, Ltd., 2009), pp. 173-187.

${ }^{53}$ J. L. Lumley and G. R. Newman, "The return to isotropy of homogeneous turbulence,"J. Fluid Mech. 82, 161-178 (1977).

${ }^{54}$ K.-S. Choi and J. L. Lumley, "The return to isotropy of homogeneous turbulence," J. Fluid Mech. 436, 59-84 (2001).

${ }^{55}$ R. B. Stull, An Introduction to Boundary Layer Meteorology, Atmospheric and Oceanographic Sciences Library (Springer Netherlands, 1988).

${ }^{56}$ N. Mori, T. Suzuki, and S. Kakuno, "Noise of acoustic Doppler velocimeter data in bubbly flow, " J. Eng. Mech. 133(1), 122-125 (2007). 